\title{
ACQUAMANILI NELLA LITURGIA CRISTIANA (IV-XVI SECOLO): IL BRONZO DELLA PINACOTECA NAZIONALE DI CAGLIARI ${ }^{1}$
}

\author{
AQUAMANILES IN CHRISTIAN LITURGY (4th TO 16th CENTURIES): \\ THE BRONZE IN THE NATIONAL GALLERY OF CAGLIARI
}

\author{
DAmiano ANEDDA, ANDREA PALA \\ Università degli Studi di Cagliari
}

\author{
Alla memoria di prof. Roberto Coroneo
}

\begin{abstract}
Riassunto: Gli acquamanili sono degli utensili che hanno una funzione liturgica nel rito cristiano, le cui origini sono verosimilmente legate a oggetti di uso profano. Il saggio analizza la funzione e le tipologie dei pochi manufatti giunti ai giorni nostri, databili tra l'VIII e il XVI secolo. Queste suppellettili occupano una posizione particolare nella storiografia specialistica, in quanto considerati dagli studiosi "tra gli oggetti più artistici", principalmente nell' ambito dei bronzi. Di tale materiale è costituito l'acquamanile a forma di pavone musealizzato nella Pinacoteca Nazionale di Cagliari, per il quale si affronta un'analisi storiografica e d'archivio, si propongono dei confronti formali e si suggeriscono un inquadramento cronologico e un ambito di provenienza basato sui dati storici.
\end{abstract}

Parole chiave: liturgia cristiana; Medioevo; Mediterraneo; bronzo; arte islamica.

\begin{abstract}
Aquamaniles are tools that have a liturgical function in the Christian rite, the origins of which are likely to be linked to objects of profane use. This essay analyses the function and the types of the few examples that have survived to this day, dating from the eighth to the sixteenth century. These ornaments have a significant position in the specialist historiography, having been considered by art historians to be "among the most artistic objects," especially those produced in bronze. Bronze is the material used for the aquamanile in the shape of a peacock that is kept in the National Gallery in Cagliari. Subjecting this important object to historiographical and archival analysis, this study advances a formal comparison that suggests a chronological framework and a place of origin based on historical data.
\end{abstract}

Keywords: Christian liturgy; Middle Ages; Mediterranean; bronze; Islamic art.

\footnotetext{
${ }^{1}$ Un particolare ringraziamento va alla Soprintendenza BAPPSAE per le province di Cagliari e Oristano, nella figura della dott.ssa Marcella Serreli, direttrice della Pinacoteca Nazionale di Cagliari, per aver agevolato il nostro lavoro di ricerca; della dott.ssa Marina Sechi, sempre disponibile per la consultazione della documentazione archivistica. La nostra riconoscenza è rivolta anche alla dott.ssa Ersilia Bussalai, direttrice della Biblioteca dello stesso Istituto, senza la quale non sarebbero emerse alcune importanti informazioni riportate nei registri inventariali. La ricerca è stata facilitata anche dalla Soprintendenza Archeologica per le province di Cagliari e Oristano, nella figura della dott.ssa Donatella Salvi, direttrice del Museo Archeologico Nazionale di Cagliari e della dott.ssa Ada Maria Opisso, responsabile dell'Archivio documenti e Fotografico, alle quali va la
} 
1. Funzione e tipologie degli acquamanili nella liturgia cristiana in epoca medievale.- 2 . L'acquamanile bronzeo della Pinacoteca Nazionale di Cagliari.- 3. Bibliografia citata.

\section{FUNZIONE E TIPOLOGIE DEGLI ACQUAMANILI NELLA LITURGIA CRISTIANA IN EPOCA MEDIEVALE ${ }^{2}$}

Il Cristianesimo annovera due importanti divisioni tra i riti: il rito orientale e il rito occidentale, entro i quali sussistono ulteriori differenze. La storia della liturgia dalle origini sino al IV secolo è sostanzialmente comune tra Oriente e Occidente, ad eccezione di elementi di culto di carattere locale che rivelano l'esistenza di diversi cerimoniali. Alla fine del IV secolo l'organizzazione della Chiesa vede il prevalere dei grandi centri storici che per tradizione cultuale emergono sulle piccole chiese, le quali assimilano particolarità liturgiche dei nuclei più ampi. Questi ultimi si impongono in virtù dei canoni sinodali e conciliari. È, infatti, nel 451 che viene convocato dall'imperatore romano d'Oriente Marciano il Concilio di Calcedonia, nel quale vengono stabiliti i quattro patriarcati di Antiochia, Alessandria, Gerusalemme, Costantinopoli e vengono annullati dallo stesso imperatore gli Atti del Concilio di Efeso del $449^{3}$. Si creano così i principali centri rituali per l'oriente cristiano, caratterizzati da un insieme di cerimonie e usi liturgici, nonché diversa lingua adottata. Tra il VI e il VII secolo in Oriente sono oramai consolidati i riti armeno, bizantino, copto e siriaco ${ }^{4}$; ognuno di questi si sviluppa sotto l'influenza del patrimonio culturale della propria "nazione" di appartenenza. Consuetudini liturgiche che si stabilizzeranno solo nel XVI secolo grazie all'utilizzo della stampa, assicurando così un ordo che sancisce i canoni e limita le molteplici varianti riportate nei manoscritti ${ }^{5}$. Diversamente in Occidente, dopo la Pace di Costantino del 313, si sviluppano due famiglie liturgiche: la gallicana e la romana ${ }^{6}$. Quest'ultima in un primo momento rimase

nostra gratitudine. Desideriamo ringraziare il professor Roberto Coroneo, docente di Storia dell'arte medievale presso il Dipartimento di Scienze Archeologiche e Storico-artistiche dell'Università di Cagliari, che fino a pochi mesi prima della sua prematura scomparsa ci ha fornito preziosi consigli per lo studio e la stesura di questo contributo.

2 Testo di Andrea Pala, email: andreapala@unica.it. Pubblicazione effettuata durante 1'attività di ricerca finanziata con le risorse del P.O.R. Sardegna F.S.E. 2007-2013 - Obiettivo competitività regionale e occupazione, Asse IV Capitale umano, Linea di Attività I.3.1. "Avviso di chiamata per il finanziamento di Assegni di Ricerca".

${ }^{3}$ P. Siniscalco, Le antiche chiese, p. 168.

${ }^{4}$ Per una sintesi sulle diverse posizioni sulla ricerca delle origini e dello sviluppo delle antiche pratiche cristiane $c f$. P.F. Bradshaw, Alle origini del culto.

${ }^{5}$ P. Amato, Note orientative, p. 21.

${ }^{6} \mathrm{Cf}$. M. Andrieu, Les Ordines romani. 
circoscritta nell'Italia centrale e nell'Africa settentrionale ${ }^{7}$, mentre quella gallicana si diffuse in tutta l'Europa occidentale, comprendendo diversi cerimoniali ${ }^{8}$, tra i quali il gallicano propriamente detto, il gotico, il celtico e l'ispanico' ${ }^{9}$. Alcune varianti del rito liturgico romano si manifestarono ad opera di ordini monastici e religiosi, come la famiglia dei benedettini dal VI-VIII secolo ${ }^{10}$ e successivamente dei francescani e domenicani dagli inizi del XIII secolo ${ }^{11}$.

Per lo svolgimento delle cerimonie sacre era utilizzata un'adeguata suppellettile liturgica che rappresenta un insieme articolato di tipologie di manufatti, la cui sistemazione scientifica è un fenomeno recente. I primi studi, effettuati in Francia, risalgono alla fine dell'Ottocento ${ }^{12}$, seguiti da quelli tedeschi degli anni Trenta del secolo successivo ${ }^{13}$; questi erano affiancati da pubblicazioni di carattere enciclopedico ${ }^{14}$, catechetico o storico liturgico ${ }^{15}$. In Italia, invece, un approccio sistematico alla materia si ebbe solo alla fine del Novecento ${ }^{16}$. Un importante contributo è stato successivamente prodotto dall'Istituto Centrale per il Catalogo e la Documentazione, afferente al Ministero per i Beni Culturali e Ambientali italiano, dai cui si trae una catalogazione che divide le suppellettili in arredi d'altare, vasi sacri, biancheria e coperture sacre, oggetti liturgici, processionali, devozionali e insegne ecclesiastiche ${ }^{17}$.

Tra gli oggetti liturgici si annoverano gli acquamanili che, come tutti gli utensili rituali cristiani, si utilizzavano all'interno degli edifici, organizzati per esprimere e favorire in tutto la comunione dell'assemblea. Nell'area del presbiterio l'azione liturgica si realizzava intorno all'altare ${ }^{18}$, nei pressi del quale venivano forse allocati anche gli acquamanili che si distinguono per materiale forma e decorazione. I materiali utilizzati si possono riconoscere nell'oro (usato soprattutto per le incrostazioni), nel bronzo e nell'argento. Quest'ultimo è documentato solo nelle fonti scritte, forse perché maggiormente soggetto ai furti, in quanto metallo "più pregiato" del bronzo. I manufatti d'argento potevano anche essere fusi per

${ }^{7}$ P. Amato, Note orientative, p. 21.

${ }^{8}$ F. Longoni, Ambrosiana. Liturgia.

${ }^{9}$ J. Pinell, Liturgia Hispánica.

${ }^{10} \mathrm{G}$. Penco, Il monachesimo .

${ }^{11}$ P. Rocha, Liturgia.

${ }^{12}$ C. Rohault de Fleury, La messe; E. Labarte, Histoires des arts; J.C. Davillier, Recherches.

${ }^{13} \mathrm{~J}$. Braun, Das christliche Altargerät.

${ }^{14}$ F. Cabrol, H. Leclerq, Dictionnaire d'archeologie.

${ }^{15}$ L.R. Barin, Catechismo; V. Casagrande, L'arte; M. Righetti, Manuale, vol. II.

${ }^{16}$ M. Fagiolo, M.L. Madonna (eds.), Roma 1300-1875.

${ }^{17}$ B. Montevecchi, S. Vasco (eds.), Suppellettile. Attualmente è on line un Thesaurus del corredo ecclesiastico di culto cattolico all'indirizzo web: http://151.12.80.76/thesaurus/home/ index.jsp [consultato: 16/02/2012], consultabile da una struttura alfabetica o gerarchica, dotata di un apparato fotografico con annessa bibliografia specialistica.

${ }^{18}$ G. Liccardo, Architettura, p.144. 
creare nuove suppellettili in sostituzione di quelle più vecchie. Sono documentati acquamanili in ceramica, emersi soprattutto dagli scavi archeologici e destinati verosimilmente alle chiese meno abbienti oppure finalizzati all'uso profano. Gli acquamanili potevano anche essere oggetti utilizzabili per le normali attività quotidiane, non necessariamente legati alla liturgia, di cui si ha testimonianza anche nella miniatura del Salterio di Bonmont (Besançon, Bibliothèque Municipale, $\mathrm{m}$. 54, c. 11v) in cui Pilato si lava le mani con un acquamanile a foggia di drago ${ }^{19}$. La maggior parte degli acquamanili superstiti è in bronzo, materiale che si ottiene con una lega di rame e stagno. Generalmente le leghe che vanno sotto il nome di bronzo hanno come componente principale il rame, lo zinco, lo stagno, tracce di piombo, di ferro, nichel, arsenico, argento e altri metalli ${ }^{20}$. Il rame, in particolare nel medio e nel tardo periodo bizantino, veniva ricavato dal metallo nativo o da ossidi, come la cuprite, oppure dal carbonato di rame (malachite) ${ }^{21}$. Ad esempio, nell'attuale Turchia e negli Urali si trovavano i principali giacimenti di rame da cui attingevano le officine costantinopolitane, mentre la cuprite è presente solo in Turchia nord orientale, nel Caucaso, Iran e Cipro ${ }^{22}$. Una forte componente di certe materie prime, come ad esempio lo zinco, può conferire alla lega un colore dorato, diversamente lo stagno dà una sfumatura rosata ${ }^{23}$. La variazione delle leghe è quindi verificabile nei lavori in bronzo eseguiti in regioni e periodi diversi, cosicché dalla loro composizione sarebbe anche possibile, seppur con cautela, trarre delle indicazioni circa l'epoca e il luogo di fabbricazione ${ }^{24}$.

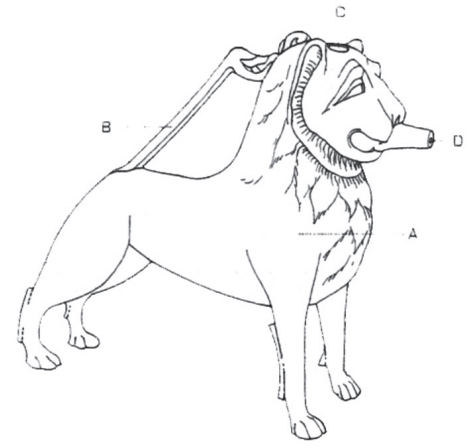

Fig. 1. Acquamanile a forma di leone, riproduzione grafica: A corpo. B impugnatura. C imboccatura. D versatoio (B. Montevecchi, S. Vasco, Suppellettile, p. 228).

\footnotetext{
${ }^{19}$ U. Mende, Acquamanile, p. 102.

${ }^{20} \mathrm{P}$. Bloch, Acquamanili, p. 115.

${ }^{21}$ F. Zagari, Approvvigionamento e lavorazione, p. 126.

${ }^{22}$ I. Baldini, L'oreficeria, p. 46.

${ }^{23}$ F. Zagari, Approvvigionamento e lavorazione, p. 127.

${ }^{24}$ P. Bloch, Acquamanili, p. 115.
} 
La struttura di queste suppellettili è distinguibile in quattro parti, denominate corpo, impugnatura, imboccatura e versatoio (fig. 1$)^{25}$. Il corpo è costituito dal nucleo centrale cavo destinato a contenere l'acqua. Questa parte definisce anche la forma dello stesso acquamanile che viene sorretto dall'impugnatura, talvolta dotata di una forma autonoma che completa il soggetto (ad esempio Sansone e il leone ${ }^{26}$. L'imboccatura è il foro che consente di rifornire l'oggetto d'acqua, che a sua volta inclinando l'acquamanile fuoriesce dal versatoio, collocato nella parte sommitale dell'oggetto. L'inclinazione non è necessaria negli acquamanili che hanno il versatoio davanti al petto, che consente il defluire dell'acqua da una bocca di scarico regolata da un rubinetto. Questa soluzione fa perdere all'impugnatura la funzione primaria di mescita dell'acqua tramite l'inclinazione ${ }^{27}$. Quando l'oggetto non era in grado di reggersi con la sola struttura del corpo, dotata di gambe o di zampe, veniva munito di un piedistallo stabilizzatore. La varietà di forme degli acquamanili è testimoniata anche nell'inventario del 1252 di St. Martin de Mayence, che restituisce:

erant uercei diversarum formarum quos manila vocant, eo quod aqua sacerdotum minibus funderetur ex eis, argentei, quidam habentes formam leonum, quedam draconu, avium et griphonum, vel aliorum animalorum quorumcumque ${ }^{28}$.

Quindi potevano avere la forma del leone, del drago, di un volatile, di un grifo o di altri animali, che comunque si riscontrano nei circa 380 acquamanili pervenuti fino ai giorni nostri, di cui 120 con le sembianze del leone, per il quale Peter Bloch ha sottolineato i significati simbolici riportati dai testi scritti $^{29}$.

Gli acquamanili avevano una forma molto varia che andava da queste appena citate sino a personaggi a cavallo, teste umane, grifoni, centauri, sìmurgh ${ }^{30}$, arpie e ancora altre forme (fig. 2).

\footnotetext{
${ }^{25}$ S. Montevecchi, S. Vasco (eds.), Suppellettile, vol. I, p. 227.

${ }^{26}$ Nell' acquamanile bronzeo custodito presso il Kunstgewerbermuseum di Berlino il corpo è costituito dal leone, sul quale si appoggia l'impugnatura sotto forma di Sansone; $c f$. G. Swarzenski, Samson.

${ }^{27}$ P. Bloch, Acquamanili, p. 116

${ }^{28}$ S. Montevecchi, S. Vasco (eds.), Suppellettile, vol. I, p. 228, con bibliografia precedente.

${ }^{29}$ P. Bloch, Acquamanili, p. 115.

${ }^{30}$ Il sīmurgh (o senmurv) è un animale della mitologia iranica la cui iconografia si diffuse anche nell'Occidente europeo. Generalmente è riconoscibile dalla testa canina, le ali di uccello con zampe artigliate e la coda di pavone. $C f$. M. Compareti, The So-called Senmurv; P. Berlekamp, From Iraq, pp. 75, 77, fig. B
} 


\begin{tabular}{|l|l|l|l|l|l|l|l|}
\hline \multicolumn{2}{|c|}{ ACQUAMANILI } \\
\hline ZIPOLOGIA & \multicolumn{2}{|c|}{ FORMA } & $\begin{array}{l}\text { Lervo/ } \\
\text { associato ad } \\
\text { altri animali/ } \\
\text { Leone } \\
\text { associato a } \\
\text { figure umane } \\
\text { Daino/ } \\
\text { Gazzella } \\
\text { Cavallo e } \\
\text { cavaliere }\end{array}$ & Cane & Ariete & Femmina di zebù & \\
\hline Antropomorfo & Busto & $\begin{array}{l}\text { Donna e } \\
\text { uomo }\end{array}$ & & & & & \\
\hline Ornitomorfo & Falco & Gallo & Oca & Pavone & & & \\
\hline $\begin{array}{l}\text { Animali } \\
\text { leggendari }\end{array}$ & Drago & Grifone & Unicorno & $\begin{array}{l}\text { Centauro/ } \\
\text { Sirena }\end{array}$ & simurgh & Arpia/ Sfinge & Fenice \\
\hline
\end{tabular}

Fig. 2. Tabella delle tipologie di acquamanili.

Questi soggetti, presi singolarmente o "appaiati”, costituivano un repertorio figurativo dal quale potevano attingere i committenti che affidavano le loro scelte alle fucine guidate da abili maestri, operanti anche nelle officine renane dei secoli XII-XIII, dalle quali si diffuse presto in tutta Europa questo straordinario oggetto di arte suntuaria, grazie alla fioritura dell'arte del bronzo iniziata sotto Carlo Magno con le porte della cattedrale di Aquisgrana ${ }^{31}$. Infatti, è nella regione della Mosa e del Reno, a Stolberg, Acquisgrana, Altenberg e Dinant $^{32}$ che nacquero, forse a partire dal X secolo, le prime fonderie di lavorazione del bronzo, sorte nei luoghi in cui si coltivavano la giallamina (lapis calaminaris) e il rame, materie prime che servivano in grande quantita ${ }^{33}$. Era dunque necessario avere le cave a breve distanza dalle officine, concentrate in un'area delimitata tra la Mosa e la Bassa Sassonia ${ }^{34}$.

Nella liturgia cristiana l'acquamanile era verosimilmente legato al rito del lavabo delle mani, che si differenzia dal pedilavium (lavaggio dei piedi) e dal capilavium, cioè il lavaggio del capo praticato nel battesimo, nella messa e in particolari funzioni come quella del Giovedì Santo ${ }^{35}$, per i quali probabilmente veniva utilizzata una brocca per le abluzioni oppure una ciotola, come quella conservata nel Museo Archeologico Nazionale di Ca-

${ }^{31}$ P. Bloch, Acquamanili, p. 8.

${ }^{32}$ Poiché molti degli acquamanili occidentali furono realizzati a Dinant, sono stati comunemente chiamati "dindanderie" cf. P. Bloch, Acquamanili, p. 115.

${ }^{33}$ P. Bloch, Acquamanili, p. 115.

${ }^{34}$ Ibidem.

${ }^{35}$ Cf. S.M. Mesina, L'acqua santa, pp. 5-50, con bibliografia precedente; N. Bux, M. Loconsole, I Misteri; G. Cremascoli, La simbologia, con bibliografia precedente; si veda anche Discussione sulla lezione Cremascoli. 
gliari ${ }^{36}$. Il rito del lavabo nella liturgia cristiana è assimilato alla purificazione e trae legittimazione dall'Esodo, in cui è riportato il momento nel quale Dio ordina a Mosè di collocare un bacile tra il tabernacolo e l'altare, dove Aronne e i figli avrebbero dovuto lavarsi le mani e i piedi prima di accostarsi alla mensa (Es 30,18; 39; 40). I riti purificatori si svolgevano presso l'altare anche per mezzo dell'acquamanile con il quale si versava l'acqua sulle mani, che defluiva in un apposito contenitore fisso detto piscina, generalmente collocato sopra una colonna o entro una nicchia ${ }^{37}$. A questo proposito Guglielmo Durando nel XIII secolo riporta: Prope altare etiam, quod Christum significat, collocatur piscina seu lauacrum, id est Christi misericordia, in qua manus lauantur ovvero "presso l'altare, che è Cristo, è posta una vasca o lavacro, cioè la misericordia di Cristo, nella quale vengono lavate le mani" ${ }^{38}$. Durante il lavabo delle mani l'acqua poteva confluire anche in un bacile concavo, già documentato nelle fonti del IV secolo ${ }^{39}$. Questo oggetto, legato alla cerimonia dell'abluzione, non si differenziava nella forma da analoghe suppellettili ad uso profano, dalle quali si distingueva per le decorazioni di simbologia cristiana eseguite sul fondo e sulla tesa ${ }^{40}$.

Anche Innocenzo III (1198-1216) nel De sacro Altaris Misterio riporta il momento dell'abluzione della mani (de ablutione manuum) che avviene prima della celebrazione della messa solenne, dopo la recitazione dei salmi: il pontefice calza i caligae (calzari) e i sandali, segue la preparazione al Vangelo e quindi si lava le mani fisicamente per pulire l'atto spiritualmente, utilizzando le parole del salmista: lavabo inter innocentes manus meas, et circumdabo altare tuum, Domine, cioè "laverò nell'innocenza le mie mani, e starò intorno al tuo altare, o Signore (Sal 26,6)"41. Lavate le mani il ministro indossa l'ammitto, che viene disposto sulle spalle e legato con due fettucce al petto $^{42}$.

I termini utilizzati nelle fonti scritte per indicare gli acquamanili potevano essere diversi, tra essi si annovera urceus, urceolus, vas manuale e

\footnotetext{
${ }^{36}$ S. Sangiorgi, L'iconografia, p. 114, con bibliografia precedente.

${ }^{37}$ S. Montevecchi, S. Vasco (eds.), Suppellettile, vol. I, p. 227.

${ }^{38}$ S. Della Torre, M. Marinelli (eds.), Rationale, pp. 24-25.

${ }^{39}$ L. Duchesne (ed.), Le Liber, p. CXLIV.

${ }^{40}$ S. Montevecchi, S. Vasco (eds.), Suppellettile, vol. I, p. 229.

${ }^{41}$ S. Fioramonti (ed.), Innocenzo III, pp. 79-85.

${ }^{42}$ L'ammitto è un fazzoletto di tela rettangolare che si avvolge intorno alla nuca, dopo il XII secolo si usava decorare il paramento sull'orlo superiore con una ricca bordura di ricami d'oro o con un gallone di stoffa preziosa, chiamato parura, plaga, aurifrisium, frisium. Questa foggia andò in disuso nel secolo XVI $c f$. M. Righetti, Manuale, vol. I, pp. 474-476; C. Callewaert, De amictu.
} 
manile per tutti i tipi di recipiente che potevano essere semplici o figurati ${ }^{43}$. Nel Liber Pontificalis è attestato un aquamanilis argenteus che viene donato da Innocenzo I (401-417) alla basilica dei Santi Gervasio e Protasio a Roma ${ }^{44}$, successivamente intitolata a San Vitale ${ }^{45}$. L'oggetto citato nella fonte del V secolo era verosimilmente una suppellettile ecclesiastica d'argento destinata a contenere dell' acqua per lavarsi le mani durante lo svolgimento liturgico, che confluiva in diversi riti non identificabili dalla descrizione del solo manufatto nel documento. In un inventario del 1227 relativo ai libri, agli argenti e agli arredi sacri delle chiese di Santa Gilla, San Pietro e Santa Maria di Cluso, già ubicate a Santa Gilla nell'areale cagliaritano, vengono elencati unu par ciminilium argenteorum ${ }^{46}$, specificazione che è verificabile dalla traduzione di Du Cange di ciminile, cioè vas in quo sacerdos sacra facies manus abluit ${ }^{47}$ e che potrebbe corrispondere a due acquamanili d'argento ${ }^{48}$, a un acquamanile e un bacile, oppure a una coppia di gemellion.

Gli acquamanili, destinati a contenere acqua, apparvero in Occidente, in Russia e nel mondo islamico in un periodo non precisabile. Nel primo caso gli oggetti giunti a noi sono databili ai secoli XII e XV-XVI, nell'ultimo risalgono anche all'VIII secolo ${ }^{49}$. Allo stato degli studi sembra che la fonte di ispirazione per tutti gli acquamanili conosciuti sia riconducibile ai modelli islamici, che pare giungessero in Occidente in grande quantità. Questa serie di oggetti prodotti in Oriente verosimilmente ebbe come tramite i modelli dell'arte sasanide poi passati all'Islam ${ }^{50}$. Tra i vasi metallici islamici si annovera la brocca di Marwān (fig. 3), conservata nel Museum of Islamic Art del Cairo e databile all'VIII secolo, che ha il versatoio a forma di gallo e appartiene a un gruppo di opere analoghe, tutte con versatoi ornitomorfi ${ }^{51}$.

Il leone è una figura molto comune negli acquamanili islamici, come ad esempio il bronzo ${ }^{52}$ fatimide con iscrizione cufica, conservato nello Staatliche kunstsammlungen di Kassel, che ha delle similitudini con

${ }^{43}$ U. Mende, Acquamanile, p. 102.

${ }^{44}$ L. Duchesne (ed.), Le Liber, p. 221.

${ }^{45}$ M.G. Zanotti, Ss. Gervasius.

${ }^{46}$ A. Capra, Inventari, p. 424.

${ }^{47}$ C. Du Cange, Ciminile.

${ }^{48}$ A. Pala, Paramenti, p. 384, con bibliografia precedente.

${ }^{49}$ U. Mende, Acquamanile, p. 102.

${ }^{50} \mathrm{D}$. Shepherd, Two silver.

${ }^{51}$ U. Scerrato, Metalli, p. 15.

${ }^{52}$ Generalmente si ritiene che la maggior parte dei manufatti islamici in metallo sia realizzata in bronzo, ma in realtà si tratta di oggetti in ottone. $C f$. R. Ward et al., Veneto Saracenic Metalwork. 


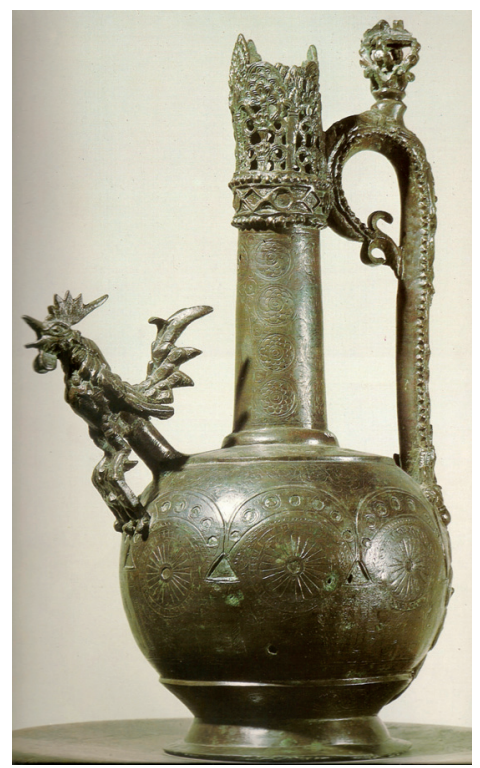

Fig. 3. Il Cairo, Museo d'Arte Islamica, brocca di Marwān (A. Papadopulo, L'Islam, fig. 77).

due leoni "occidentali", uno dei quali conservato a Oslo e ritenuto del XII secolo, mentre il secondo è stato acquisito dal Nationalmuseet di Copenaghen (fig. 4) ${ }^{53}$.

È stato notato che questo tipo di bronzi piuttosto che acquamanili erano pertinenti a fontane: oggetti che imitavano leoni di maggiori dimensioni raffigurati nei palazzi, associati anche ad altri tipi di animali, secondo un uso che ha radici profonde nel Vicino Oriente ${ }^{54}$. Tra i più antichi acquamanili orientali conosciuti si annovera una serie di manufatti ornitomorfi conservati all'Hermitage, tra i quali spicca quello a forma di gallo non datato e probabilmente "condizionato" dall'arte sasanide ${ }^{55}$. Entro la produzione islamica è possibile citare anche un acquamanile a forma di falcone conservato nel monastero di Santa Caterina del Sinai (fig. 5), per il quale è stata proposta una datazione all'VIII o al IX secolo ${ }^{56}$. La fabbricazione di tali oggetti nei primi secoli dell'Islam ha suscitato interrogativi sulla loro origine, come ad esempio

\footnotetext{
${ }^{53}$ U. Mende, Acquamanile, p. 106.

${ }^{54}$ E. Cruikshank Dodd, On a bronze.

${ }^{55}$ J. Orbeli, Sassanian.

${ }^{56}$ E. Baer, Metalwork, pp. 189-190.
} 


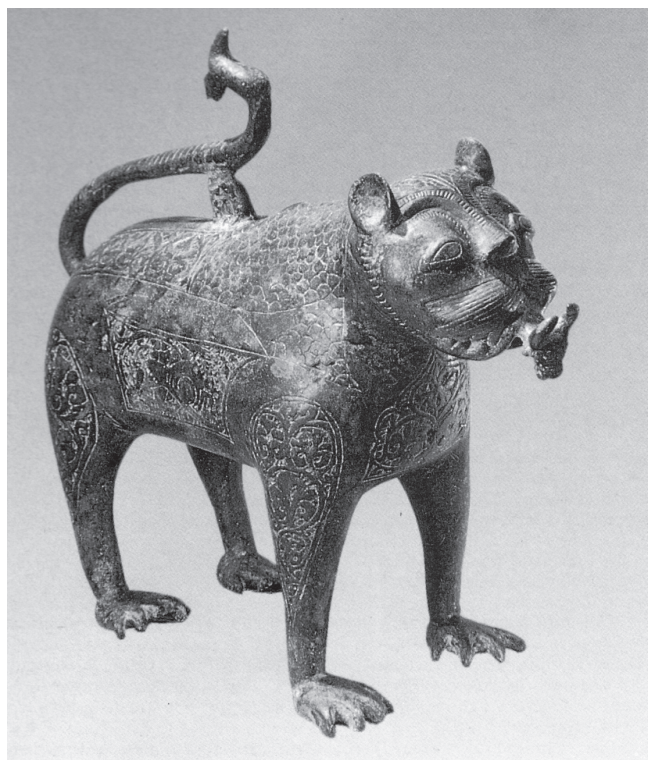

Fig. 4. Copenaghen, Nationalmuseet, acquamanile e forma di leone, bronzo (U. Mende, Acquamanile, p. 106).

per il volatile bronzeo ora conservato a Berlino nel Staatliche Museen-Museum für Islamische Kunst e ascritto al VIII-X secolo (fig. 6).

A questo proposito Oleg Grabar si chiede se questi utensili non fossero il risultato di imitazioni di ori o argenti realizzati per la corte dei prìncipi della dinastia omayyade o di quella abbaside, oppure riflessi della nuova committenza cittadina (la classe media mercantile del mondo musulmano) della superiore arte dei prìncipi ${ }^{57}$. I contatti tra l'arte orientale e occidentale sarebbero documentabili dall' acquamanile a forma di pavone, realizzato da 'amal 'abd al-malik al-nasrani cioè "opera di un servitore di re cristiano" e conservato nel Museo del Louvre (fig. 7) ${ }^{58}$. Ma uno dei primi esempi di acquamanile probabilmente prodotto in Occidente si trova nel Victoria and Albert Museum. L'oggetto è realizzato in bronzo dorato, placcato in argento e decorato a niello ${ }^{59}$, per il quale è

${ }^{57}$ O. Grabar, Arte islamica, pp. 232-234.

${ }^{58}$ S. Makariou, Aquamanile, p. $111, \mathrm{n}^{\circ} 87$.

${ }^{59}$ Il niello era costituito da una mistura di solfuri metallici che apportava una forte nota di nero o di grigio scuro. Questa amalgama si applicava nelle depressioni ottenute sul metallo per mezzo di strumenti diversi e l'utilizzo di tecniche orafe particolari. Il trattato De Diversibus Artibus, redatto nell'XI secolo dal monaco Theophilus, riporta un'accurata descrizio- 


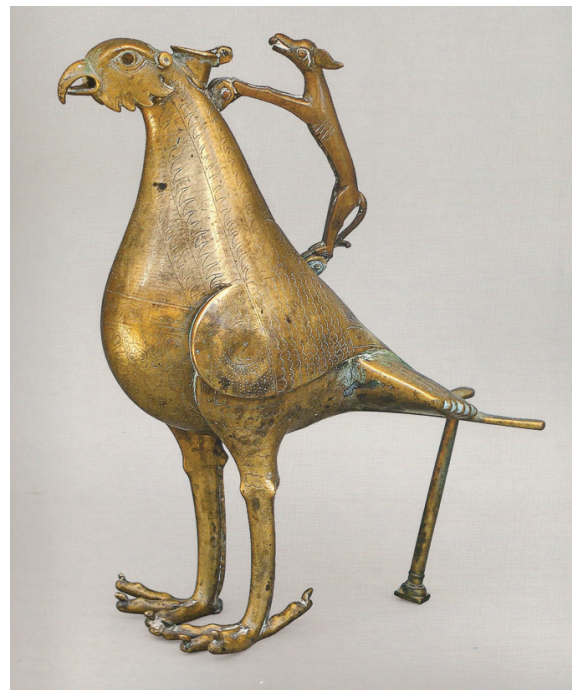

Fig. 5. Monte Sinai, Monastero di Santa Caterina, falco, bronzo

(H.C. Evans, B. Ratliff, Byzantium and Islam, p. 65, $\left.\mathrm{n}^{\circ} 38\right)$.

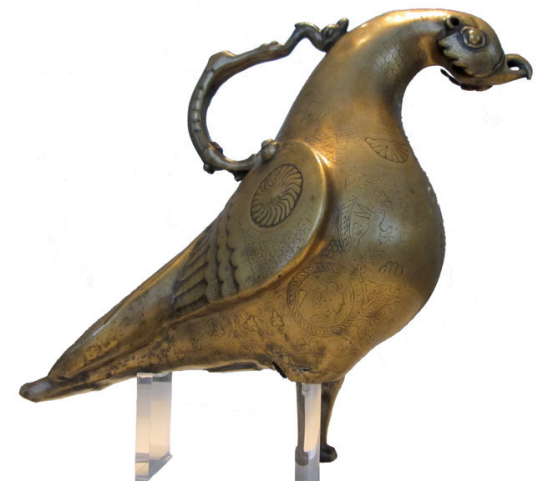

Fig. 6. Berlino, Staatliche Museen-Museum für Islamische Kunst, volatile (bruciaprofu$m i$ ?), bronzo (foto D. Anedda).

stata proposta la fabbricazione nel territorio della Mosa nei primi anni del XII secolo (fig. 8$)^{60}$.

Si realizzavano molte opere di questo genere anche nelle fonderie della Bassa Sassonia, da cui forse proviene un cavaliere della Collezione Carrand al Museo del Bargello di Firenze. Gli acquamanili venivano prodotti anche nella Germania del nord, in particolare a Hildesheim e a Lubecca. Numerosi manufatti sono attribuibili al XIII e XIV secolo, fra cui si annoverano un leone conservato al Louvre e un esemplare conservato al Metropolitan $\mathrm{Mu}$ seum (fig. 9), attribuibili al XIII secolo ${ }^{61}$. Nel secolo successivo si ricerca una tendenza decorativa maggiore che si raggiunge nelle forme slanciate del XV secolo, riscontrabili nell'esemplare di Cluny ${ }^{62}$ (fig. 10).

ne dell'utilizzo di questo materiale e del metodo di realizzazione dei decori. In particolare nel Libro III si descrive la realizzazione di un calice a due manici. Cf. R. Hendrie, An essay, pp. 234-237 (cap. XXVIII), 238-239 (cap. XXIX), 242-243 (cap. XXXII), 256-257 (cap. XLI).

${ }^{60} \mathrm{U}$. Mende, Acquamanile, p. 105.

${ }^{61}$ P. Bloch, Acquamanili, p. 116.

${ }^{62}$ Ibidem. 


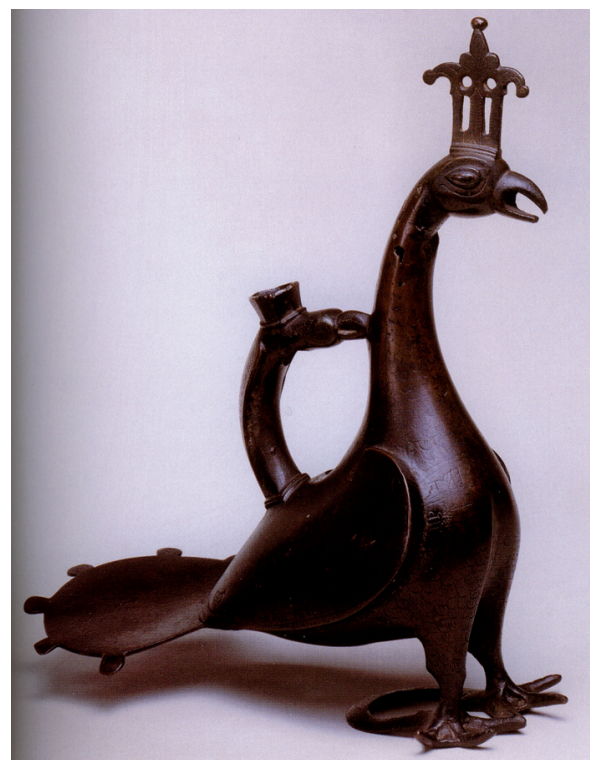

Fig. 7. Parigi, Musée du Louvre, acquamanile a forma di pavone, bronzo, h. $39,5 \mathrm{~cm}$

(M. Bernus-Taylor, Les Andalousies, p. 111, n० 87).

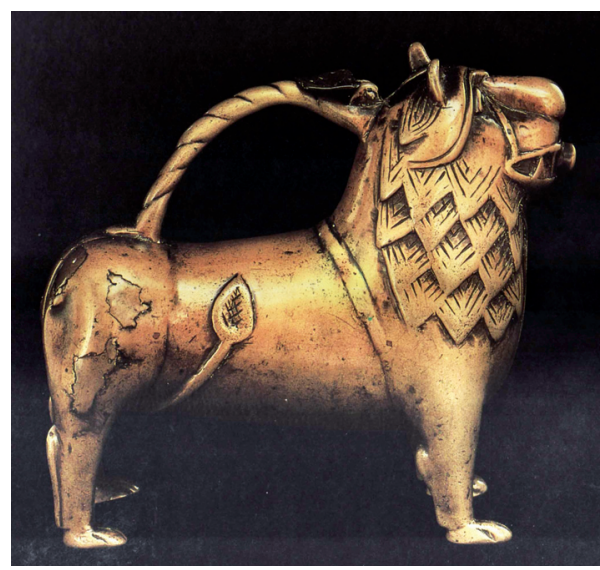

Fig. 9. New York, The Metropolitan Museum of Arts, "dono di Irwin Untermyer", acquamanile a forma di leone, $\mathrm{h} .19,7 \mathrm{~cm}$. (P. Bloch, Acquamanili, p. 17, tav. 2 sp.)

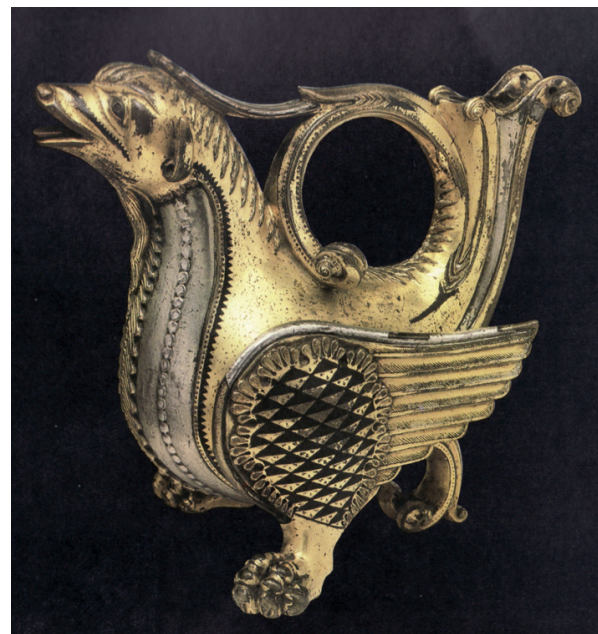

Fig. 8. Londra, Victoria and Albert Museum, acquamanile a forma di drago, damaschinato in argento e dorato, niello, h. $18.3 \mathrm{~cm}$ (P. Bloch, Acquamanili, p. 18, tav. 22 sp.)

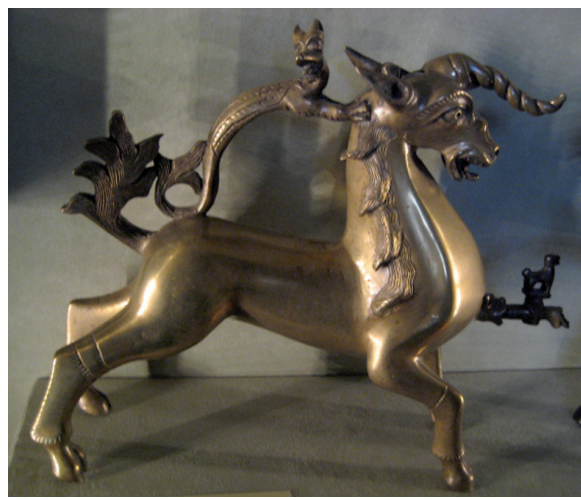

Fig. 10. Parigi, Musée National du Moyen Age - thermes de Cluny, acquamanile a forma di unicorno (foto A. Pala) 
Come visto, la maggior parte di queste suppellettili é decontestualizzata, spesso giunta alle raccolte museali dai tesori delle chiese o per vie diverse non sempre tracciabili. L'acquamanile della Pinacoteca di Cagliari (fig. 11) fu ritrovato nella località di San Salvatore presso Mores (Sassari), acquisito nel $1919^{63}$ e confluito nella Pinacoteca Nazionale di Cagliari, nel cui registro inventariale gli è stato attribuito il numero $13^{64}$.

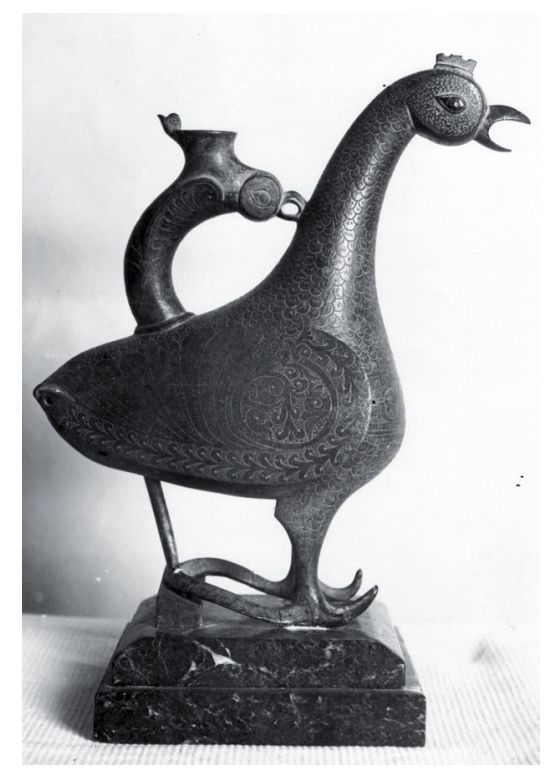

Fig. 11. Cagliari, Pinacoteca Nazionale, acquamanile a forma di pavone, bronzo, h. $28 \mathrm{~cm} \mathrm{x} 1$. $32 \mathrm{~cm}$, (Archivio Fotografico Soprintendenza BAPPSAE per le province di Cagliari e

Oristano, Acquamanile e bronzi: (CA) Pinacoteca, manufatti in bronzo, statue e vetrine sezione busti, sezione cornici, foto $\left.\mathrm{n}^{\circ} 3324\right)$.

Nel 1980 l'acquamanile è documentato nell'elenco del materiale cultoreo custodito all'interno della Pinacoteca Nazionale di Cagliari ${ }^{65}$. L'oggetto è stato recentemente sottoposto a expertise ${ }^{66}$, ma non si è ancora sciolto

${ }^{63}$ L'acquisto è segnalato da L. Siddi, Acquamanile; M. Serreli, Acquamanile.

${ }^{64}$ Pinacoteca Nazionale, Registro inventario 10-Sculture (SC), p. 2.

${ }^{65}$ Archivio Soprintendenza BAPPSAE per le province di Cagliari e Oristano, Cagliari (CA), Pinacoteca Nazionale opere mobili, Cagliari Pinacoteca, Inventario opere mobili, Biblioteca (13-9-80), $n^{\circ} 1$ acquamanile bronzeo a forma di pavone.

${ }^{66} \mathrm{La}$ valutazione dell'oggetto è stata richiesta dai funzionari della Soprintendenza Ersilia Bussalai e Maria Passeroni al Departement des Arts de L'Islam del Musee du Louvre, nella figura di Rachel Beaujean-Deschamps, la quale attribuisce al bronzo un valore di 1.500.000,00 di 
il quesito sul ritrovamento occasionale a Mores della suppellettile, forse già dotazione di una chiesa importante oppure "proprietà" di un prelato di alto livello. A questo proposito potrebbe essere interessante valutare il carattere itinerante del vescovo della sede episcopale di Sorres nel XV secolo ${ }^{67}$, da cui la parrocchiale di Mores dipendeva ${ }^{68}$. Il vescovo sorrano Ardizzone nel 1432 possedeva una casa a Borutta ${ }^{69}$ e un palatiu in hue faghimus recidentia in sa çitade de Sasseri ${ }^{70}$, ovvero un palazzo dove il prelato risiedeva nella città di Sassari. La notizia che sembra utile portare all'attenzione è che nel 1442, quando forse è vescovo Sancio $^{71}$, si manifestava da parte dell'ecclesiastico la volontà di migliorare la casa di Borutta ${ }^{72}$. Con questo scopo si decise di vendere al pievano di Mores una domo coperta et domos disfatas et ortu su quale sy clamat Piscopia, atto di vendita stipulato nella chiesa di Santa Caterina di Mores $^{73}$. Tra l'altro, pur nelle scarse notizie sulle rendite vescovili della fine del XV secolo, si ha l'impressione che esse non dovessero essere particolarmente floride. In questo periodo emerge un comportamento fiscale "dispotico" nei confronti dei preti da parte del vescovo, il quale pretendeva il versamento di un diritto come cattedratico: su cabrevadu ${ }^{74}$. In sintesi dalla documentazione si evince che nel XV secolo a Mores vi erano dei possedimenti del vescovo di Borutta, il quale concluse una vendita di beni immobili al pievano di Mores. Si deduce inoltre una precaria situazione economica della diocesi sorrana nello stesso arco cronologico. Queste condizioni avrebbero potuto favorire anche l'alienazione di arredi sacri in possesso del vescovo, al fine di averne un ritorno economico ${ }^{75}$. Allo stato degli studi questa rimane solo un'ipotesi non suffragata da prove documentarie. Tantomeno è possibile conoscere con certezza l'originale allocazione dello straordinario oggetto d'arte suntuaria oggi nella Pinacoteca Nazionale di Cagliari.

euro. $C f$. Biblioteca Soprintendenza BAPPSAE, e-mail cartacea del 31 luglio 2006 Da: Rachel. Beajeaun-Dechamps@louvre.fr A: ersilia.bussalai@beniculturali.it,oggetto: acquamanile.

${ }^{67} \mathrm{R}$. Turtas, Il registro, p. XII.

${ }^{68}$ G. Zichi, Sorres, pp. 150, 302.

${ }^{69} \mathrm{R}$. Turtas, Il registro di Sorres, p. XII, nota 13.

${ }^{70}$ S.S. Piras, G. Dessì, Il registro di San Pietro, p. 48, doc. 120.

${ }^{71} \mathrm{R}$. Turtas, Il registro di Sorres, p. XII, nota 13.

${ }^{72}$ S.S. Piras, G. Dessì, Il registro di San Pietro, p. 89, doc. 225.

${ }^{73}$ Ibidem.

${ }^{74} \mathrm{R}$. Turtas, Il registro di Sorres, p. XII.

${ }^{75}$ A. Pala, Arredo, pp. 133-134. 


\section{L'ACQUAMANILE BRONZEO DELla PinACOTECA NAZIONALE Di CAGLIARI ${ }^{76}$}

L'acquamanile cagliaritano ${ }^{77}$ ha la forma di un pavone e misura 32 centimetri di altezza e 28 di lunghezza (fig. 12). È ornato con piume stilizzate marcate con motivi a squame, al cui interno sono incise brevi linee parallele fra loro. Le ali sono costituite da una serie di lunghe penne, otto in un lato e undici nell'altro, decorate con motivi a spina di pesce. Nella parte anteriore delle ali un ramo avvolge decorazioni ad arabesco e a foglie arricciate. Dallo stesso tralcio deriva una fascia che delimita le ali, ornata da una serie continua di semipalmette.

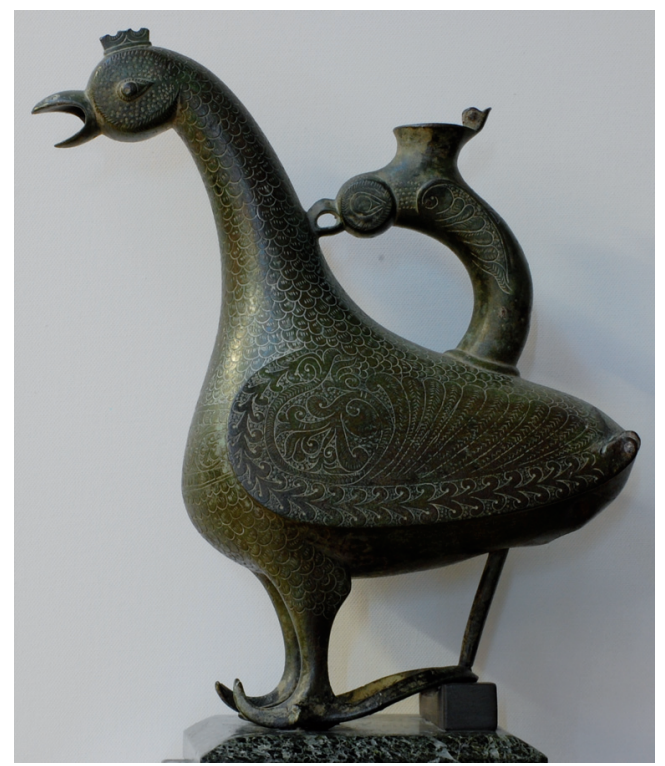

Fig. 12. Cagliari, Pinacoteca Nazionale, acquamanile a forma di pavone, bronzo (foto D. Anedda, A. Pala).

Il petto del volatile è attraversato da una banda con motivi a girali che contengono foglie pentalobate. Al di sopra, entro un clipeo con motivi

\footnotetext{
${ }^{76}$ Testo di Damiano Anedda, email: damianoanedda@gmail.com.

${ }^{77}$ Le immagini dell' acquamanile bronzeo di Cagliari sono state riprodotte su concessione del Ministero per i Beni e le Attività Culturali - Direzione Regionale per i Beni Culturali e Paesaggistici della Sardegna - Soprintendenza per i Beni Architettonici il Paesaggio il Patrimonio Storico Artistico ed Etnoantropologico per le province di Cagliari e Oristano: prot. 7575 del 18.04.2011, class. 28.13.10/2.2.
} 
fitomorfi arricciati, è incisa una croce potenziata a bracci patenti (fig. 13). La stessa è replicata nella parte superiore dell'impugnatura, costituita dalla testa e dal collo di un uccello (fig. 14).Le superfici del corpo prive di decorazione, quali testa e parti di impugnatura, petto e ali, sono ornate da piccoli cerchi con punto centrale. Sul manico è presente l'imboccatura, dal diametro di 3,5 x 4,5 centimetri, per l'inserimento dell'acqua che fuoriusciva dal becco del volatile. Sulla stessa apertura sono visibili i raccordi del coperchio andato perduto.

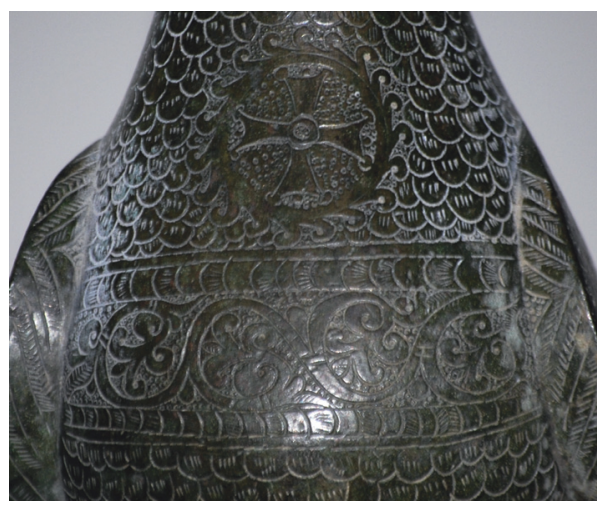

Fig. 13. Cagliari, Pinacoteca Nazionale, acquamanile a forma di pavone, petto, bronzo (foto D. Anedda, A. Pala).

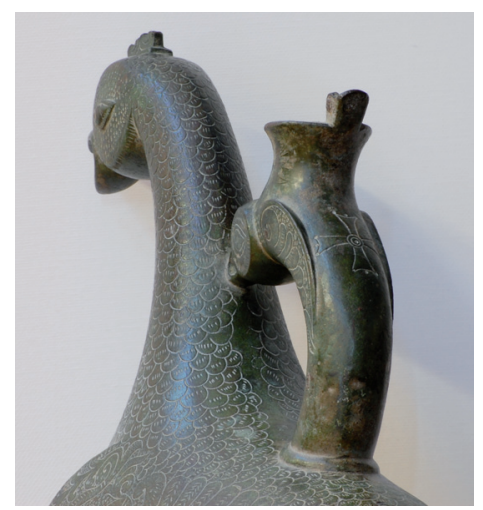

Fig. 14. Cagliari, Pinacoteca Nazionale, acquamanile a forma di pavone, impugnatura, bronzo (foto D. Anedda, A. Pala).

Sono scomparsi anche il pennacchio e la coda, di cui permangono i punti di giunzione. In posizione posteriore, sotto i raccordi a cui era fissata la coda, è visibile un piccolo foro circolare dal diametro di 1,7 centimetri, la cui funzione non è chiara. Le zampe sono unite tra loro mediante un anello stabilizzatore sul quale è fissata un'asticella che si congiunge con la parte posteriore del pavone. Questo elemento è simile a quello del volatile bronzeo custodito presso il monastero di Santa Caterina del Sinai ${ }^{78}$ (fig. 5).

Benché il manufatto sia entrato a far parte della collezione museale cagliaritana nel $1919^{79}$, esso ha suscitato l'attenzione della critica solo a partire dagli anni cinquanta del ventesimo secolo, quando fu presentato a Parigi in occasione dell'esposizione sull' arte medievale in Italia del 1952. Nel catalogo

\footnotetext{
${ }^{78}$ A. Ballian, Bird-shaped vessel; A. Ballian, A Singular Gift.

${ }^{79} \mathrm{Cf}$. nota 63 .
} 
della mostra l'acquamanile cagliaritano è collocato tra i bronzi di produzione araba e messo in relazione per stile e decori al grifo bronzeo pisano ${ }^{80}$. Umberto Scerrato nel 1966 incluse il pavone nella monografia dedicata ai metalli islamici, considerandolo opera arabo-andalusa del XII secolo ${ }^{81}$. Renata Serra ha analizzato il manufatto in diverse occasioni, ritenendolo non di manifattura araba ma prodotto in ambiente legato a Bisanzio, perché il motivo della croce clipeata presente nel petto del volatile sarebbe da attribuire a tale contesto ${ }^{82}$. La stessa studiosa, in un articolo dedicato all'oratorio delle anime di Massama (OR) e ai suoi rapporti con l'architettura ispanica d'epoca visigotica, ha ritenuto le decorazioni a girali presenti nella banda incisa sul petto del volatile simili a quelle di alcune brocche visigotiche e ai motivi vegetali presenti nei fregi decorativi degli edifici ispanici dello stesso periodo ${ }^{83}$. Nel 1976 Serra riteneva inoltre l'acquamanile dall'armonia compositiva talmente sicura da non potersi attribuire ad artefice islamico, ma realizzato da un artigiano bizantino di stretta osservanza classicista ${ }^{84}$. Successivamente la studiosa ha ritenuto il manufatto fuso da un bronzista di cultura islamica nella bottega di un centro cristiano $^{85}$. L'analisi di Marcella Serreli parte dalle analogie tra gli acquamanili di Cagliari e del Louvre. La studiosa ritiene questi manufatti realizzati nel XII secolo nella penisola iberica, prodotti in ambito culturale islamico e utilizzati anche dalle comunità cristiane ${ }^{86}$. Le ricerche di Anna Contadini, in accordo con la tesi di Scerrato nel ritenere il bronzo della Pinacoteca Nazionale di Cagliari di provenienza ispanica ${ }^{87}$, lo datano ai secoli XI-XII e collegano l'opera sia al pavone del Musée du Louvre ${ }^{88}$ (fig. 7), sia all'acquamanile bronzeo custodito presso la David Collection di Copenaghen ${ }^{89}$ (fig. 15). Secondo Contadini i tre bronzi sono accomunati dalla stessa stilizzazione di occhi, becco, cresta, e lo stesso senso di rigidità e monumentalità ${ }^{90}$. La studiosa ha avvalorato in diverse occasioni la provenienza ispanica di sculture bronzee zoomorfe quali il grifo pisano ${ }^{91}$, l'acquamanile di Cagliari e il quadrupede

\footnotetext{
${ }^{80}$ La scheda relativa all'acquamanile cagliaritano ("Aquamanile en forme de colombe") verosimilmente fu stilata da Raffaello Delogu, nominato in una nota che elenca gli autori delle schede del catalogo. $C f$. Trésors d'art, s.p.

${ }^{81}$ U. Scerrato, Metalli p. 80, $\mathrm{n}^{\circ} 34$.

${ }^{82}$ C. Maltese, R. Serra, Episodi, p. 190.

${ }^{83}$ R. Serra, L'oratorio, pp. 53-54.

${ }^{84}$ Eadem, Le raccolte, s.p.

${ }^{85}$ Eadem, Pittura, p. 19.

${ }^{86}$ M. Serreli, Acquamanile.

${ }^{87}$ A. Contadini, Acquamanile.

${ }^{88}$ S. Makariou, Aquamanile, p. 111, n ${ }^{\circ} 87$.

${ }^{89}$ Eadem, Aiguière.

${ }^{90}$ A. Contadini, Acquamanile.

${ }^{91}$ Cf. A. Contadini, R. Camber, P. Northover, Beasts that roared.
} 
del Museo Nazionale del Bargello ${ }^{92}$. Questi bronzi, a suo avviso, presentano caratteristiche ornamentali che richiamano l'organizzazione dell'apparato decorativo utilizzato nei tessuti, formato da bande dotate anche di iscrizioni, che fungono da settori delimitanti per le diverse zone della scultura ${ }^{93}$.

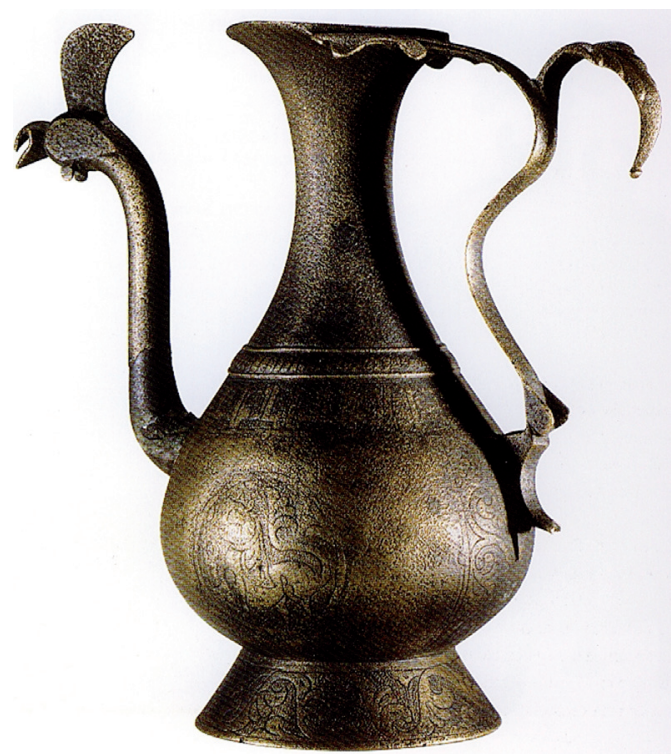

Fig. 15. Copenaghen, David Collection, acquamanile in bronzo (M. Bernus-Taylor, Les Andalousies, fig. 168).

Roberto Coroneo in un primo momento ha ritenuto il manufatto fuso in ambiente italo-bizantino, a Venezia o in Sicilia ${ }^{94}$; successivamente ha suggerito come luogo di produzione l'Andalusia o le terre che la Reconquista restituiva via via al processo di ricristianizzazione della Penisola iberica ${ }^{95}$.

L'analisi dell'acquamanile cagliaritano, così come quella delle sculture bronzee zoomorfe d'epoca medievale, mette in luce diverse problematiche, tra le quali l'individuazione dei luoghi di produzione, riconosciuti dalla critica in zone geograficamente molto distanti tra loro, dall'Iran alla penisola iberica, e dei canali attraverso i quali i manufatti sarebbero giunti

\footnotetext{
${ }^{92}$ A. Contadini, Quadrupede.

${ }^{93}$ Eadem, Le arti in Spagna, p. 58.

${ }^{94} \mathrm{R}$. Coroneo, Acquamanile.

${ }^{95} \mathrm{Idem}$, Il reliquiario, p. 125.
} 
nei luoghi in cui sono attualmente custoditi. Relativamente alla funzione originaria e alle successive destinazioni d'uso di alcuni di questi manufatti, Anna Contadini ha formulato alcune ipotesi convincenti ${ }^{96}$, in particolare sul grifo pisano ${ }^{97}$, il leone della collezione Mari-Cha ${ }^{98}$ e il volatile di San Frediano a Lucca ${ }^{99}$ (fig. 16).

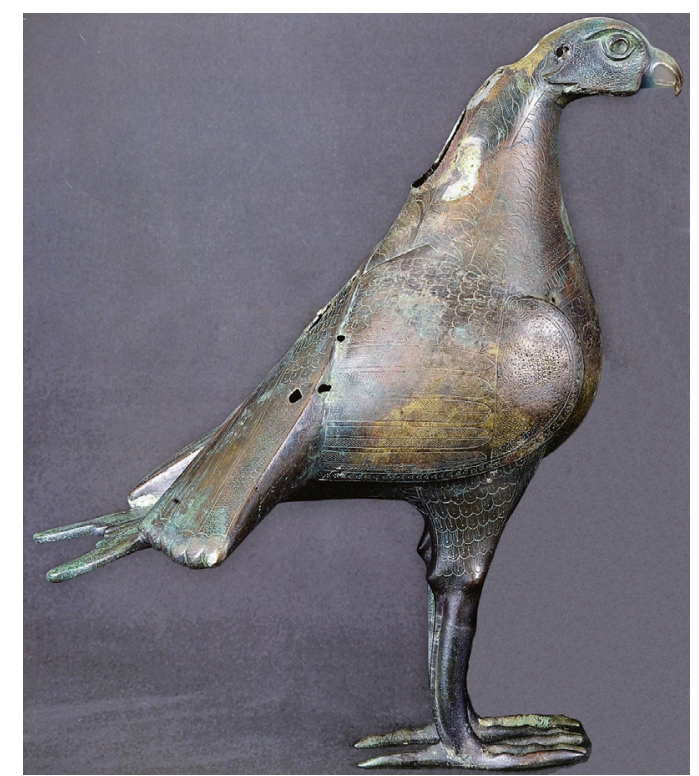

Fig. 16. Lucca, San Frediano, volatile (C. Baracchini et al., Lucca, p. 198).

Alcuni bronzi zoomorfi d'epoca medievale sono strettamente legati alla storia del collezionismo ${ }^{100}$, come il leone ritrovato a Monzón de Campos (Palencia) e oggi al Louvre ${ }^{101}$, appartenuto al pittore Mariano Fortuny i Marsal, uno tra i più prolifici collezionisti ottocenteschi di arte arabo-andalusa. Carlos Navarro ha recentemente curato un saggio sull'inventario dei beni di Fortuny, dal quale si evince che tra gli oggetti della sua collezione spiccava il

\footnotetext{
${ }^{96}$ A. Contadini, Translocation and transformation, pp. 53-57.

${ }^{97}$ C. Robinson, Grifo.

${ }^{98}$ A. Contadini, R. Camber, P. Northover, Beasts that roared; C.T. Little, Leone

${ }^{99}$ R. Silva, La Basilica di San Frediano, pp. 223-226, figg. CXIV-CXVI; R. Ward, Acquamanile.

${ }^{100}$ M.G. Stasolla, Il collezionismo.

${ }^{101}$ M. Gómez-Moreno, El arte árabe, fig. 396a.
} 
leone di Monzón ${ }^{102}$, riconoscibile anche in una fotografia dello studio romano del pittore catalano ${ }^{103}$ (fig. 17).

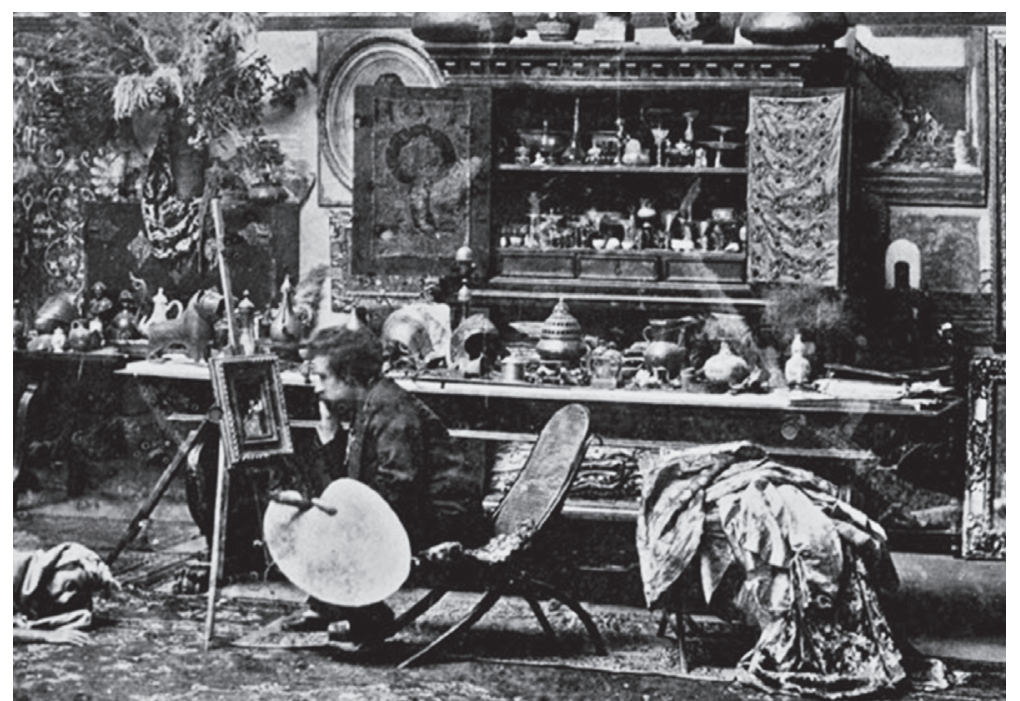

Fig. 17. Roma, Atelier del pittore Mariano Fortuny i Marsal, fotografia 1873-1874 (C. González, Testamentaría, fig. 4).

Per lo studio del manufatto custodito nella Pinacoteca Nazionale di Cagliari, può essere utile considerare che nei secoli della formazione dell'arte islamica l'area iranica fu uno dei centri più produttivi di manufatti metallici, grazie alla continuità con la tradizione toreutica del periodo sasanide ${ }^{104}$. Questa zona geografica, insieme alla Transoxiana, era famosa per la produzione di rame ${ }^{105}$, componente principale del bronzo ${ }^{106}$. Sono presenti numerose miniere di rame anche nel Maghreb, mentre la produzione di stagno risulta più povera, tanto che le manifatture mediorientali si servivano di stagno malese ed europeo ${ }^{107 .}$ In Iran lo stile e le tipologie sasanidi furono imitati dalla scuola

${ }^{102}$ C. González Navarro, Testamentaría, fig. 4.

${ }^{103}$ M.G. Stasolla, Il collezionismo, p. 680.

${ }^{104}$ G. Curatola, G. Scarcia, Le arti, p. 147.

${ }^{105}$ M. Bernardini, Bronzo, p. 782.

${ }^{106} \mathrm{Cf}$. nota 19.

${ }^{107}$ M. Bernardini, Bronzo, p. 782. 
dei bronzisti del Khorasan ${ }^{108}$, che comprendeva anche il Mavera al-Nahr ${ }^{109}$. Le tecniche di lavorazione dei metalli di questa tradizione, rimasta attiva dall'VIII al XIX secolo ${ }^{110}$, si diffusero anche in Iraq, dove si formò la scuola di Mossul ${ }^{111}$.

Ai bronzi relativi all'Egitto fatimide ${ }^{112}$ si ascrivono sculture zoomorfe quali il cervo ${ }^{113}$, il leone ${ }^{114}$ e la lepre ${ }^{115}$ custoditi presso il Museo di arte islamica del Cairo, come pure i cervi del Museo di Capodimonte a Napoli $^{116}$ e dello Staatliches Museum für Völkerkunde di Monaco di Baviera ${ }^{117}$. Al-Andalus, in particolare nel periodo califfale, fu un centro attivo nella produzione di manufatti in metallo, in alcuni casi documentati in ritrovamenti archeologici. Ne sono testimoni le lampade bronzee provenienti dalla moschea di Medina Elvira (Atarfe, Granada). Queste lampade, recuperate e pubblicate nel 1888 da Gómez-Moreno González ${ }^{118}$ e in seguito da suo figlio Gómez-Moreno Martínez ${ }^{119}$, sono custodite presso il Museo Arqueológico y Etnológico di Granada. Altri manufatti bronzei andalusi di particolare interesse sono alcuni bruciaprofumi ${ }^{120} \mathrm{e}$ varie lucerne ${ }^{121}$ provenienti da Cordova e Granada. A questi si aggiunge un cofanetto in argento commissionato dal califfo al-Hakam II per suo figlio Hisham, custodito presso il tesoro della cattedrale di Girona ${ }^{122}$. Per l'analisi delle sculture zoomorfe arabo-andaluse al momento ci si basa su dati archeologici non perfettamente documentati, che tuttavia consentono di attribuire numerosi manufatti alla Spagna dei secoli X-XII. Valga come esempio il già citato leone bronzeo custodito al Louvre, ritrovato tra le rovine di una fortezza islamica dell'XI secolo situata a Monzón de Campos, nei pressi di Palencia ${ }^{123}$. Questi dati permettono di

\footnotetext{
${ }^{108}$ Per i bronzi del Khorasan $c f$. A.S. Melikian-Chirvani, Les bronzes, 1974-1978.

${ }^{109}$ M. Bernus-Taylor, Oggetti, p. 374.

${ }^{110}$ Ibidem .

${ }^{111}$ U. Scerrato, Arte islamica, p. 541; sui bronzi della scuola di Mossul $c f$. A. Ballian, Three medieval Islamic brasses.

${ }^{112}$ Per un quadro generale sull'Egitto fatimide si rimanda al colloquio: Barrucand, Marianne (ed.), L'Égypte fatimide.

${ }^{113}$ M. Abbas, The Fatimids, p. 76, $\mathrm{n}^{\circ} 66$.

${ }^{114}$ J. Bloom, Arts of the City Victorious, pp. 97-99, fig. 68.

${ }^{115}$ J. Allan, W. Ezzy, J. Scarce, Metalwork, p. 166, n 169.

116 A. Contadini, Le arti del periodo, p. 137.

${ }^{117}$ A. Von Gladiss, Figure de cerf.

${ }^{118}$ C. Vílchez (ed.), Las lámparas.

${ }^{119}$ M. Gómez-Moreno, El arte, pp. 324-325, figg. 385-386.

${ }^{120}$ Ibidem, p. 335, figg. 395a-b.

${ }^{121}$ Ibidem, p. 335, figg. 392-393.

${ }^{122}$ M. Casamar, Arqueta.

${ }^{123}$ M. Charritat, Lion.
} 
considerare di derivazione andalusa una serie di sculture bronzee zoomorfe che la tradizione storiografica degli inizi del XX secolo riteneva di produzione fatimide ${ }^{124}$.

Per l'esame dell'acquamanile ritrovato a Mores possono essere utili le testimonianze di storici vissuti durante o successivamente al califfato di Cordova, come Al-Maqqari, Ibn Hayyan e Ibn Bashkuwal. Al-Maqqari, scrittore originario di Tlemcen vissuto a cavallo tra i secoli XVI e XVII, in un'opera dedicata alla storia delle dinastie arabe in Spagna riporta diversi episodi ripresi dallo storico cordovese Ibn Hayyan. Tra le meraviglie di Madinat alZahra quest'ultimo riferisce di due fontane che costituivano l'ornamento di maggior pregio del palazzo. Una di esse fu trasportata da una località orientale non specificata. Nella fontana furono incastonate perle e pietre preziose e la stessa fu decorata con dodici figure in oro rosso realizzate a Cordova. Queste ultime, probabilmente fuse in bronzo e successivamente dipinte in oro, rappresentavano diversi animali, quali un leone con ai lati un'antilope e un coccodrillo, un'aquila, un drago, una colomba, un falco, un pavone, una gallinella, un gallo, un nibbio e un avvoltoio ${ }^{125}$. Ibn Bashkuwal, erudito conoscitore della storia di al-Andalus vissuto a Cordova nel XII secolo, riferisce che nei palazzi della capitale califfale l'acqua sgorgava dalla bocca di diversi animali realizzati in pietra, marmo e metallo ${ }^{126}$.

L'analisi delle fonti suggerisce una prolifica produzione di raffinati bronzi zoomorfi e ornitomorfi nella capitale del califfato d'occidente, che presumibilmente erano impiegati non solo per una loro applicazione nelle fontane, ma anche per un utilizzo più ampio, come suppellettili di vario genere, utilizzabili anche come doni ufficiali.

Il pavone cagliaritano presenta una chiara somiglianza con due manufatti bronzei ritenuti di lavorazione islamica: il pavone conservato presso la Furusiyya Arts Foundation a Vaduz ${ }^{127}$ (fig. 18) e quello del Louvre ${ }^{128}$ (fig. 7). Il bronzo della Pinacoteca Nazionale è privo di pennacchio e coda, componenti presenti nell'acquamanile del Louvre. Quello di Vaduz invece conserva solo il pennacchio. Un altro elemento che associa i tre pavoni è l'anello stabilizzatore che unisce le zampe, comune anche ad acquamanili bronzei realizzati in luoghi geograficamente distanti (fig. 19) rispetto a quello cagliaritano ${ }^{129}$.

\footnotetext{
${ }^{124}$ E. Kühnel, Maurische, fig. 124; G. Migeon, Manuel, vol. I, p. 277, fig. 184.

${ }^{125}$ P. de Gayangos (ed.), The history, p. 236.

${ }^{126}$ L. Torres Balbás, Arte, p. 747.

${ }^{127}$ S. Makariou, Aquamanile, p. $112, \mathrm{n}^{\circ} 88$.

${ }^{128}$ Eadem, Aquamanile, p. 111, $\mathrm{n}^{\circ} 87$.

${ }^{129} C f$. l'acquamanile riportato nel catalogo J.M. Rogers, The Arts, pp. 78-79, $\mathrm{n}^{\circ} 68$.
} 


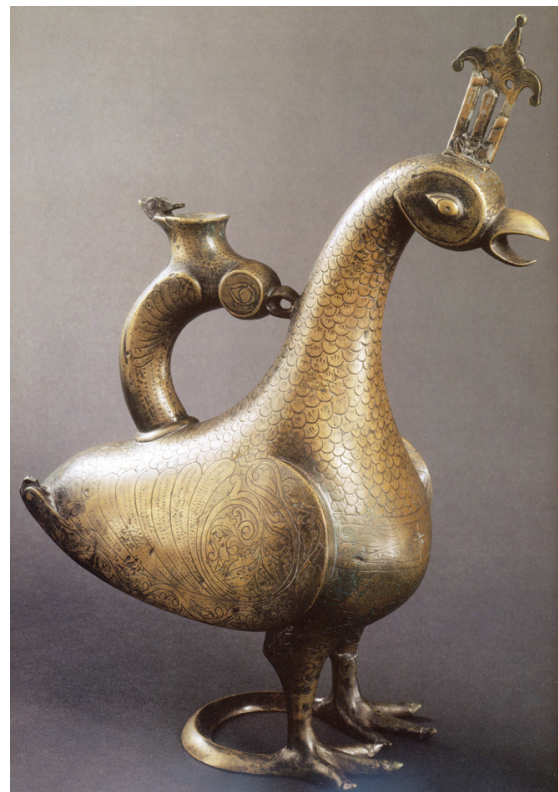

Fig. 18. Vaduz, Furusiyya Arts Foundation, acquamanile a forma di pavone, bronzo (M. Bernus-Taylor, Les Andalousies, fig. 88).

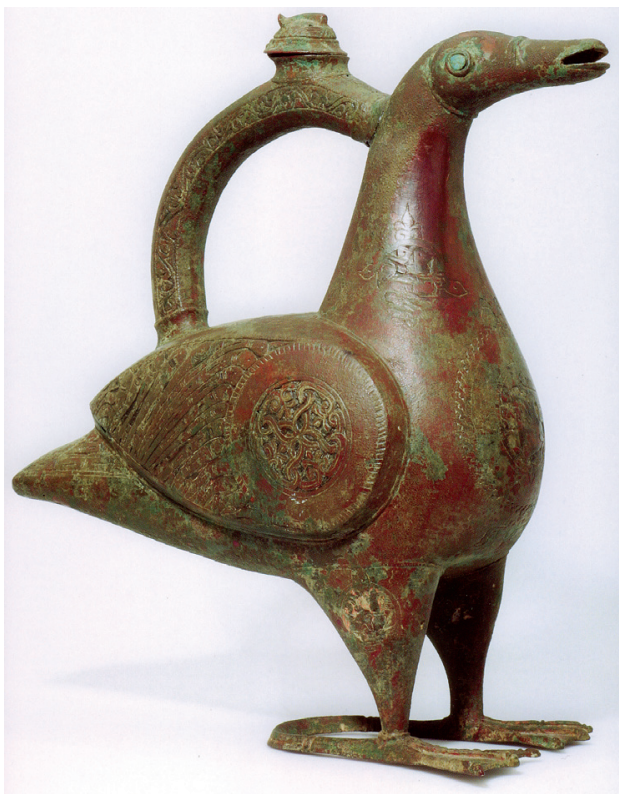

Fig. 19. Nasser D Khalili Collection, acquamanile a forma di volatile, bronzo (J.M. Rogers, The Arts, fig. 68).

Per lo studio del bronzo di Cagliari è opportuno un approfondimento sull'acquamanile del Louvre, il quale ha suscitato l'interesse della critica per le due iscrizioni incise sul petto, la superiore in latino e l'inferiore in arabo. La prima, preceduta da una croce, recita: OPUS SALOMONIS ERAT. Il nome Salomone in essa presente potrebbe riferirsi al re profeta biblico ${ }^{130}$. Lo storico ottocentesco Longperier interpreta la parte in latino dell'iscrizione come un riferimento a un prodotto realizzato con grande abilità (Opus Salomonis $)^{131}$.

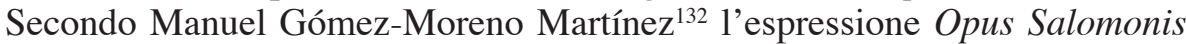
è invece da riferire ai manufatti realizzati con la tecnica della fusione a cera persa $^{133}$.

${ }^{130}$ O. Grabar, About a bronze, p. 121.

${ }^{131}$ A. de Longperier, Vase, p. 359.

${ }^{132}$ M. Gómez-Moreno, Iglesias, p. 388.

${ }^{133}$ Per la tecnica della fusione a cera persa $c f$. H. Maryon, La lavorazione, pp. 213-228. 
Robert-Henri Bautier ha avanzato una proposta su datazione e provenienza del manufatto del Louvre, basata su una diversa interpretazione dell'iscrizione latina.

Se questa epigrafe indicasse il nome dell'artefice del pavone, a suo avviso, al posto dell'imperfetto erat dovremmo aspettarci est o più semplicemente opus Salomonis con il verbo sottinteso. Bautier dopo la T di ERAT individua una lettera $X$, proponendo la seguente revisione, preceduta da un signum crucis: OPUS. SALOMONIS. ERA TX.

Per giustificare questa proposta lo studioso francese riferisce che nella paleografia ispanica del periodo che intercorre tra gli anni 1000 e 1120, la lettera $\mathrm{T}$ era utilizzata al posto della cifra romana $\mathrm{M}$. Nel caso dell'iscrizione del pavone del Louvre, aggiungendo la X alla T si otterrebbe la data 1010. La numerazione dell'era ispanica ebbe inizio nel 38 a.C., anno della conquista romana della penisola iberica. Tale calendario è stato universale in Spagna fino al pieno medioevo. L'associazione TX corrisponderebbe dunque all'anno 972. Questa interpretazione confermerebbe sia la provenienza ispanica sia la data di realizzazione dell'acquamanile del Louvre. Secondo Bautier la cifra 972 risulta inoltre essere coerente con le lettere irregolari dell'iscrizione latina, attribuibili al X secolo o alla prima metà dell'XI ${ }^{134}$. Già Manuel Gómez-Moreno Martínez fece notare che un'iscrizione simile a quella latina presente nel petto del pavone parigino, sia nel contenuto sia perché anch'essa è preceduta da una croce, è incisa in una lucerna bronzea conservata al Museo Arqueológico Nacional di Madrid ${ }^{135}$. In essa si legge: OC OPUS SALOMONIS ERAT ${ }^{136}$. Per quanto riguarda l'iscrizione araba dell'acquamanile del Louvre, essa recita عمل عبد الملك النصرني ('ammal 'abd al-malik al-nasrani) e si può tradurre: “opera di Abd al-Malik il Cristiano". L'espressione 'abd al-malik potrebbe anche corrispondere a quella latina Servus Domini ${ }^{137}$. In questo caso si dovrebbe tradurre "opera di un servitore di re cristiano".

Oleg Grabar si è chiesto se il termine "Salomone", presente nelle iscrizioni latine dell'acquamanile e della lucerna sopracitati, riguardi il nome del loro artefice o indichi piuttosto un oggetto degno del re biblico, in linea con l'espressione "Opus Salomonis" utilizzata in epoca medievale per segnalare l'alta qualità di un manufatto ${ }^{138}$. Grabar si sofferma anche sull'iscrizione araba, riconoscendo che la sua peculiarità non riguarda tanto l'informazione che tramanda, irrilevante per un acquirente "latino". Piuttosto l'esotismo un

\footnotetext{
${ }^{134}$ R.H. Bautier, Datation, p. 95.

${ }^{135}$ M. Gómez-Moreno, Iglesias, pp. 387-389.

136 J. Zozaya, Lamp.

${ }^{137}$ S. Makariou, Aquamanile, p. 111, $n^{\circ} 87$.

${ }^{138}$ O. Grabar, About a bronze, p. 121.
} 
po' misterioso di questa epigrafe avrebbe suscitato un grande interesse nei confronti di un compratore di lingua non araba. Lo stesso studioso afferma che le due iscrizioni dell'acquamanile del Louvre possono essere interpretate in sei modi differenti ${ }^{139}$, fra i quali ne predilige due: A work of Solomonic (beauty), year 962, made by Abd al-Malik the Christian, ovvero "un lavoro di bellezza salomonica, anno 962, realizzato da Abd al-Malik il Cristiano"; It was (or could have been) a work (worthy of) Solomon (but or and?) it was made by Abd al-Malik the Christian ${ }^{140}$, ovvero "questo fu un lavoro (degno) di Salomone ma/e fu eseguito da Abd al-Malik il Cristiano".

Non è del tutto chiara la funzione degli acquamanili di Cagliari, Parigi e Vaduz. Questi possono essere stati richiesti da un committente cristiano o fusi per poi essere venduti a un simile personaggio. Secondo Grabar, poiché forma e decorazione dei manufatti non implicano usi cerimoniali o liturgici specifici, è possibile che siano stati realizzati in funzione di un ampio mercato, senza particolari vincoli di carattere religioso ${ }^{141}$. La presenza di una croce incisa nel petto dei tre pavoni sarebbe un dato da tenere in considerazione. Il simbolo cristiano, infatti, potrebbe sia indicare un ambito di produzione dei manufatti circoscritto ad ambiente cristiano piuttosto che arabo, sia suggerire che gli acquamanili sarebbero stati acquistati per essere utilizzati come suppellettili liturgiche all'interno di un edificio religioso.

Nello studio sull'acquamanile parigino Bautier afferma che il luogo di produzione di questi manufatti è da ricercare nella penisola iberica, non però in zone governate dai cristiani, quali Asturie, Leon o Navarra. In questo caso il bronzista non avrebbe utilizzato per sé l'appellativo "il Cristiano", come riporta l'iscrizione araba del pavone del Louvre. Tale appellativo sarebbe invece ragionevole se utilizzato in al-Andalus, per la presenza di diverse comunità di mozarabi nelle città di Cordova, Siviglia e Toledo ${ }^{142}$. Oleg Grabar, pur concordando con Bautier sul fatto che la T finale dell'epigrafe corrisponda alla cifra romana $\mathrm{M}$, non si trova d'accordo con lo studioso francese nell'individuare la lettera $\mathrm{X}$ successiva alla lettera T. Lo stesso Grabar data infatti l'acquamanile del Louvre all'anno 962, cifra dedotta dal fatto che l'era ispanica ebbe inizio a partire dal 38 a.C. Lo studioso ipotizza che l'acquamanile del Louvre possa essere una testimonianza dell'arte mozarabica, i cui contenuti e usi sono legati alla cristianità ma le forme sono associate al mondo islamico ${ }^{143}$. Grabar evidenzia inoltre che lo stile dell'epigrafe latina dell' acquamanile e la comparazione di questa con l'iscrizione

${ }^{139}$ Ibidem, pp. 122-123.

${ }^{140}$ Ibidem, pp. 123-124.

${ }^{141}$ Ibidem, p. 120.

${ }^{142}$ R.H. Bautier, Datation, p. 98.

${ }^{143}$ O. Grabar, About a bronze, p. 124. 
della lucerna del Museo Arqueológico Nacional di Madrid ${ }^{144}$ rendono ammissibile l'origine iberica del manufatto parigino, affermando che quest'ultimo sarebbe stato eseguito per un contesto commerciale in cui i rapporti culturali o etnici erano meno significativi del giudizio sulla qualità dell'oggetto ${ }^{145}$.

Avinoam Shalem ha ipotizzato che una serie di manufatti eburnei e bronzei risalenti ai secoli XII-XIII, tra cui annovera il pavone di Cagliari, possano esser stati realizzati da artigiani arabi convertiti alla fede cristiana, attivi in Spagna, Sicilia o sud Italia ${ }^{146}$. Lo studioso ritiene che diversi manufatti islamici d'epoca medievale furono utilizzati in ambito cristiano senza essere trasformati, lasciando intatte persino le iscrizioni in lingua araba eventualmente presenti in essi. Queste, a suo avviso, sarebbero state interpretate dai possessori degli oggetti come antiche iscrizioni ebraiche o lettere usate nei primi secoli del Cristianesimo. Le stesse iscrizioni arabe avrebbero conferito al manufatto un sapore orientale e dunque più vicino alla Terra Santa ${ }^{147}$. Shalem ritiene inoltre che tali oggetti durante il medioevo sarebbero stati accolti nei tesori delle chiese, sia perché eseguiti da botteghe tecnologicamente avanzate, sia perché legati al più alto livello sociale dell'epoca, poiché realizzati per re o alti dignitari di corte. Generalmente i tesori delle chiese occidentali accoglievano manufatti islamici realizzati con materiali preziosi quali avorio, argento, cristallo di rocca e pietre preziose. Gli oggetti islamici eseguiti in materiale non prezioso come il bronzo erano accettati nei tesori o utilizzati come suppellettili liturgiche solo se la loro tecnica esecutiva era superiore a quella degli altri prodotti disponibili sul mercato. Il caso di un acquamanile (in arabo $i b r \bar{\imath} k)^{148}$ bronzeo come quello cagliaritano può essere perfettamente incluso all'interno di questa riflessione. Shalem conclude affermando che, in epoca medievale, i manufatti riferibili all'ambito islamico non erano probabilmente considerati dalla committenza ecclesiastica relativi a una religione rivale e antagonista, ma semplicemente realizzati in un' atmosfera culturale differente ${ }^{149}$.

L'interpretazione di Bautier relativa alla datazione dell'acquamanile del Louvre, basata sull'iscrizione latina, è da ritenersi coerente solo in parte. La lettera $\mathrm{X}$ finale individuata dallo studioso non è visibile chiaramente e pertanto non risulta probante. Accettare che la cifra $\mathrm{T}$ dell'iscrizione di questo manufatto sia un'indicazione cronologica induce a considerare coeva all'acquamanile la lucerna bronzea del Museo Arqueológico Nacional di Madrid,

144 J. Zozaya, Lamp.

${ }^{145}$ O. Grabar, About a bronze, p. 124.

${ }^{146}$ A. Shalem, Islam Christianized, p. 99.

${ }^{147}$ Ibidem, p. 137.

${ }^{148}$ E. Baer, Ibrīk.

${ }^{149}$ A. Shalem, Islam Christianized, p. 173. 
che riporta un'epigrafe essenzialmente identica a quella del pavone parigino. Datare due manufatti della seconda metà del X secolo allo stesso anno risulterebbe essere un'ipotesi poco sostenibile. Vista la somiglianza tra gli acquamanili del Louvre e di Cagliari, tale proposta avrebbe in parte risolto anche la questione cronologica del pavone della Pinacoteca Nazionale. Sarebbe forse più prudente lasciare aperto il dibattito sulla cronologia ricavata dalle epigrafi del pavone parigino e della lucerna di Madrid.

Non è possibile stabilire se i coperchi che serravano le imboccature degli acquamanili di Cagliari, Parigi e Davos fossero decorati, poiché sono andati perduti. Si può solo ipotizzare che la loro superficie superiore fosse ornata, come nel caso dell' acquamanile della collezione Nasser David Khalili, che mostra una protome leonina sul coperchio ${ }^{150}$ (fig. 19).

Dal punto di vista formale l'acquamanile di Cagliari sembra più vicino a quello di Vaduz piuttosto che a quello del Louvre. La loro altezza (35,8 $\mathrm{cm}$ il pavone di Vaduz, $32 \mathrm{~cm}$ quello di Cagliari) varia solo perché quello della Furusiyya Arts Foundation conserva intatto il pennacchio. Il capo e il manico dei due manufatti sono particolarmente simili, così come gli attacchi della coda, del coperchio e del pennacchio. Il becco è aperto in entrambi, sia nell'impugnatura sia nella testa per la fuoriuscita dell'acqua. Gli occhi sono stati realizzati in modo equivalente, incisi nel manico e in rilievo con pupilla trapanata nel capo (fig. 12). La decorazione del corpo dei due volatili è pressoché identica, salvo alcune differenze visibili nella fascia che circonda le ali, decorata da una serie continua di semipalmette in quello cagliaritano e da un ramo ondulato con foglie in quello di Vaduz. Una differenza tra le decorazioni dei due manufatti si riscontra anche nelle bande incise sui loro petti. Se quella del bronzo cagliaritano è decorata con motivi a girali e foglie pentalobate, al centro della fascia di quello del Liechtenstein è invece presente una croce fiancheggiata da due palmette racchiuse da involucri cuoriformi, dai quali hanno origine due semipalmette (fig. 18). Per individuare confronti iconografici fra motivi decorativi che ornano manufatti realizzati con modalità artistiche distinte tra loro è necessario muoversi con cautela; tuttavia si può constatare che gli stessi elementi vegetali incisi nel petto del pavone di Vaduz sono presenti nella scultura decorativa ispanica altomedievale, con riferimenti puntuali appurabili nei plutei della chiesa di San Miguel de Escalada ${ }^{151} \mathrm{e}$ in alcuni frammenti scultorei dell'edificio asturiano di Santianes de Pravia ${ }^{152}$ (fig. 20).

${ }^{150} \mathrm{Cf}$. l'acquamanile riportato nel catalogo J.M. Rogers, The Arts, pp. 78-79, $\mathrm{n}^{\circ} 68$.

${ }^{151}$ A. Arbeiter, S. Noack-Haley, Christliche Denkmäler, figg. 80a, 81c.

${ }^{152}$ L. Caballero, et al., Las iglesias, p. 58, figg. 15, 113. 


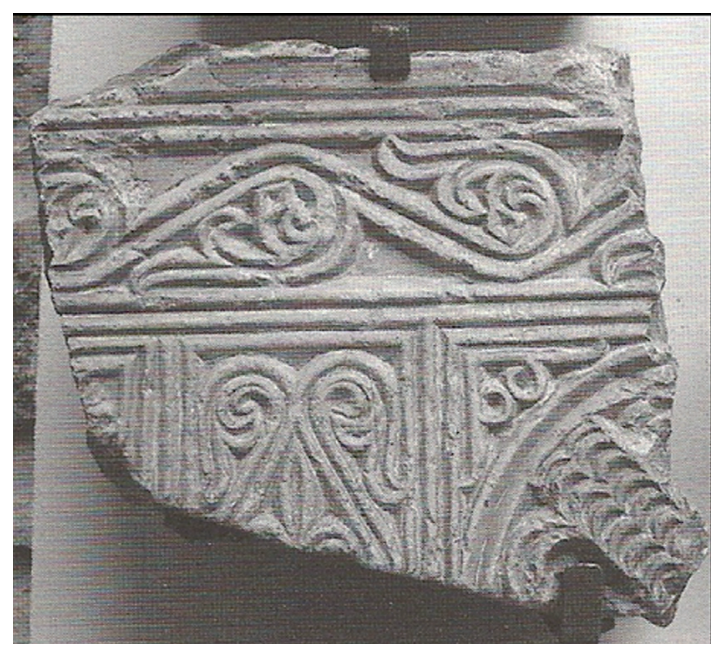

Fig. 20. Pravia (Asturias), San Juan Evangelista, frammento di pluteo (L. Caballero, et al., Las iglesias, fig. 113).

Si può chiamare in causa anche un bronzo battuto all'asta il 29 Aprile 2003 al Christie's di Londra ${ }^{153}$, citato da Rosalinda Inglisa nella sua tesi di laurea ${ }^{154}$. Si tratta di un volatile bronzeo acquisito dal Museum of Islamic Art di Doha, probabilmente usato come getto di fontana, le cui similitudini con l'acquamanile di Cagliari sono evidenti sia nelle forme plastiche del collo, della testa e del becco, sia nelle incisioni a squame ricavate nel corpo del manufatto ${ }^{155}$. Potrebbe trattarsi di uno degli esemplari di scultura zoomorfa in bronzo dorato che abbellivano i giardini della capitale del califfato occidentale, citati da al-Maqqari ${ }^{156}$, così come il quadrupede bronzeo del Museo Nazionale del Bargello, di probabile provenienza cordovese, usato in origine come getto di fontana, come suggeriscono le aperture del ventre e della bocca ${ }^{157}$.

Tornando all'acquamanile cagliaritano, la stessa soluzione del clipeo inciso nel petto del pavone è utilizzata frequentemente nella scultura zoomorfa medievale islamica, come si può riscontrare in manufatti bronzei at-

153 http://www.christies.com/presscenter/pdf/04042003/April\%202003\%20-\%20Islamic\% 20Art\%20\&\%20Manuscripts.pdf [consultato: 17-02-2012].

${ }^{154}$ R. Inglisa, Arte islamica, p. 129, fig. 71.

${ }^{155} \mathrm{Cf}$. nota 142 .

${ }^{156}$ P. de Gayangos (ed.), The history, p. 236.

${ }^{157}$ A. Contadini, Quadrupede. 
tribuiti dalla critica ad aree geografiche molto distanti tra loro, dall'Iran alla penisola iberica. Il cervo custodito presso il Museo Arqueológico Provincial di Cordova ${ }^{158}$ e il rapace del museo dell'Hermitage ${ }^{159}$ (fig. 21) sono solo due tra i numerosi manufatti nei quali è stato adottato lo stesso schema decorativo ${ }^{160}$. I motivi fitomorfi arricciati, che delimitano il disco inciso nel petto del pavone, erano utilizzati già nelle decorazioni in stucco d'epoca sasanide ${ }^{161}$ (fig. 22).

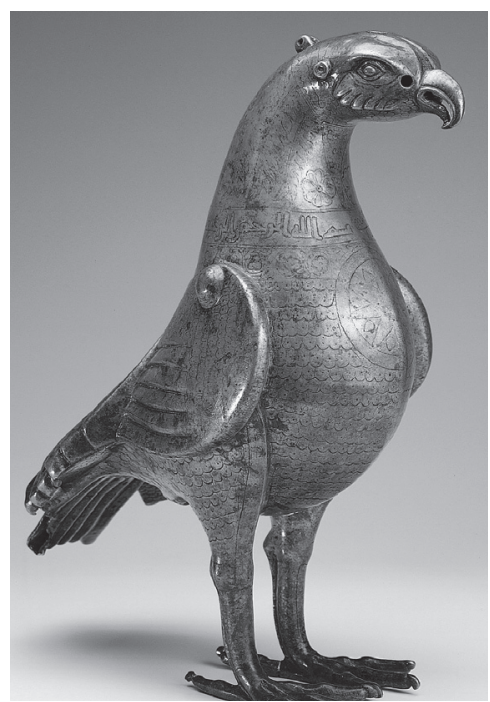

Fig. 21. San Pietroburgo, Hermitage, volatile in bronzo

(M. Bernus-Taylor, Oggetti, fig. 481).

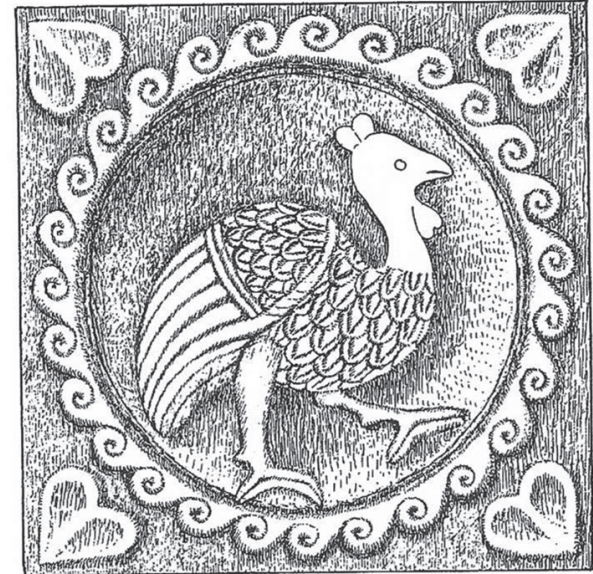

Fig. 22. New York, Metropolitan Museum of Art, placca in stucco da Ctésiphon, riproduzione grafica (J. Kröger, Sasanidischer, fig. 24).

Questi all'interno ospitavano motivi prevalentemente zoomorfi. La serie continua di semipalmette, incisa nella fascia esterna delle ali del pavone di Cagliari, sembrerebbe una mutazione in senso naturalistico dei motivi cuoriformi di tradizione orientale (fig. 23), non estranei ad alcuni manufatti scultorei altomedievali iberici ${ }^{162}$.

${ }^{158}$ S. Makariou, Bouche.

${ }^{159}$ M. Bernus-Taylor, Oggetti, p. 384, fig. 481.

${ }^{160}$ Per altri esempi $c f$. H. Glück, E. Diez, Arte, p. 710, n 429; J.M. Rogers, The Arts, pp. $78-79, \mathrm{n}^{\circ} 68$.

${ }^{161}$ J. Kröger, Sasanidischer, p. 54, fig. 24.

${ }^{162}$ D. Anedda, Arte, pp. 163-164, figg. 10, 12. 


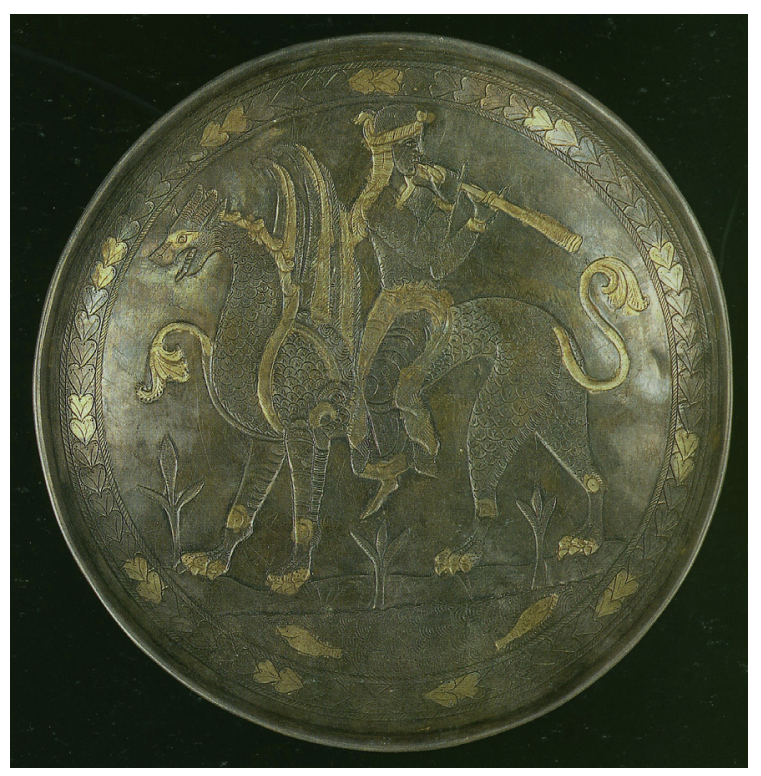

Fig. 23. San Pietroburgo, Hermitage, piatto sasanide, argento con doratura (B. Overlaet, Splendeur, fig. 73).

Le decorazioni vegetali del petto (fig. 13) e quelle ad arabesco delle ali dell'acquamanile di Cagliari (fig. 12) sono state messe in relazione con le palmette lavorate a sbalzo del cofanetto di Hisham $\mathrm{II}^{163}$. Se da un lato si può riscontrare una certa somiglianza tra gli elementi decorativi dei due manufatti, tuttavia girali e arabeschi del pavone presentano una dinamicità che conferisce loro un aspetto più naturalistico rispetto ai motivi più statici dello scrigno argenteo di Girona (fig. 24). Il dato realistico dei girali del pavone cagliaritano è accentuato dalla presenza di brevi incisioni praticate nei rami della vegetazione. Tali segni sembrano riprendere i supporti metallici utilizzati per fissare alle pareti i rami di viti o piante rampicanti $i^{164}$.

Come già osservato, alcune fonti scritte testimoniano la presenza di sculture bronzee zoomorfe utilizzate come elementi di fontana nella Cordova del X secolo. Nella capitale del califfato d'occidente si insediarono maestranze altamente qualificate, legate alla corte principesca e specializzate nella lavorazione di metalli, avorio e altri materiali. Con la fondazione di Madinat al-Zahra da parte di 'Abd al-Rahman III, insieme agli organi burocratici e am-

${ }^{163}$ A. Contadini, Acquamanile.

${ }^{164}$ D. Anedda, Bronzi islamici, p. 49. 


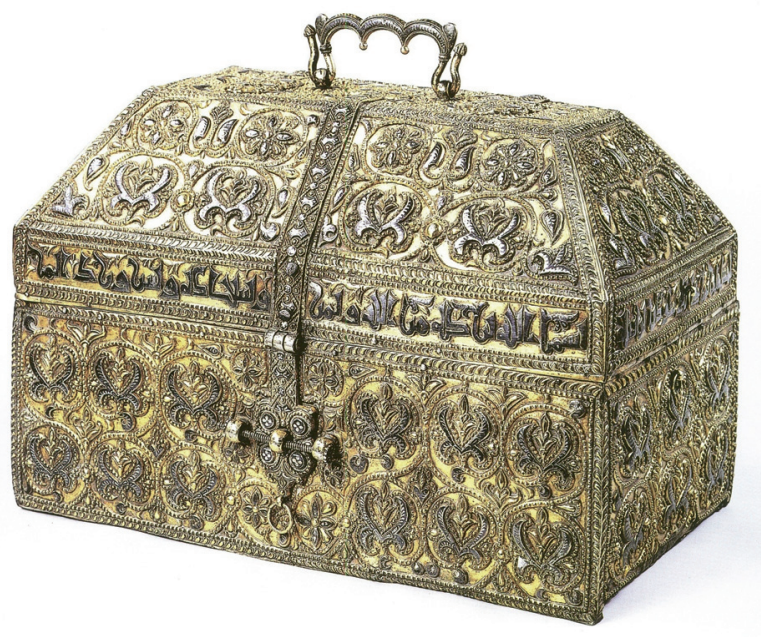

Fig. 24. Girona, Tesoro della cattedrale, scrigno in argento (J.D. Dodds, Al-Andalus, p. 209).

ministrativi statali, furono spostati nella nuova residenza palatina la zecca, la manifattura tessile reale e il centro ufficiale degli artigiani ${ }^{165}$, la cui produzione era indirizzata a soddisfare sia le richieste del califfo e della sua famiglia, sia le necessità relative ai regali diplomatici, di cui si servivano i governanti per i doni ufficiali ${ }^{166}$.

Il peso politico degli arabi di al-Andalus si attenuò in seguito alla disgregazione del califfato, avvenuta a causa di un periodo di guerra civile che, tra gli anni 1010-1013, portò all'assalto e alla conseguente distruzione di Madinat al-Zahra ${ }^{167}$. In seguito a simili avvenimenti si formarono alcuni regni, detti taifas, i più significativi dei quali ebbero come capitali Siviglia, Toledo, Saragozza e Granada. All'indebolimento politico-amministrativo degli arabi non corrispose però una decadenza nella produzione artistica, che ebbe uno slancio inaspettato nei nuovi regni grazie anche al fatto che gli artigiani della corte omayyade cordovese dovettero cercare fortuna altrove, trovandola in parte presso le corti dei muluk al-tawa'if (re delle regioni) ${ }^{168}$. È noto a tal proposito l'esempio di alcuni intagliatori d'avorio trasferitisi presso la corte toledana dei Dhu'l-Nun, che installarono nella città di Cuenca un atelier al

${ }^{165}$ A. Contadini, La Spagna, pp. 108-109.

${ }^{166}$ N. Silva, Marfiles, p. 256.

${ }^{167}$ Cf. E. Lévi-Provençal, Decadencia.

${ }^{168}$ A. Contadini, La Spagna, p. 109. 
servizio dei governanti ${ }^{169}$. È probabile che questi artigiani non badassero al fatto che i territori in cui si trasferivano fossero i nuovi regni delle taifas o le regioni riacquisite dai cristiani. È verosimile pensare che tali maestranze dovettero adeguarsi a realizzare prodotti commercializzabili in un ampio mercato, non solo locale ma rivolto ai commerci terrestri e marittimi ${ }^{170}$.

È interessante analizzare le notizie di Ibn Hayyan, che offrono importanti informazioni sulle relazioni commerciali tra penisola iberica, Sardegna e Campania. Lo storico arabo informa dell'arrivo a Cordova, in data 24 agosto del 942, di un messaggero del signore di Sardegna, giunto insieme ad alcuni mercanti amalfitani ${ }^{171}$. Questi portavano con sé le loro merci preziose, tra cui lingotti d'argento puro e broccati ${ }^{172}$. Non è questa la sede per soffermarsi sulle caratteristiche dei tessuti campani del X secolo, di cui riferisce anche Ibn Hawqal nel Libro delle vie e dei reami, ritenendo il lino prodotto a Napoli di qualità ineguagliata in nessun altro tiraz del mondo ${ }^{173}$. Corrado Zedda si è chiesto se i lingotti d'argento citati nella fonte si possano associare alla contemporanea presenza dei sardi, presupponendo un eventuale sfruttamento dei giacimenti argentiferi dell'Isola già a partire da quella data ${ }^{174}$. Nei secoli successivi (XI-XII) il mondo arabo, a causa della carenza d'argento, avrebbe persino interrotto la coniazione dei dirhams ${ }^{175}$, elemento che potrebbe avvallare l'ipotesi dello sfruttamento delle miniere d'argento sarde. Nessuna emergenza archeologica però conferma questa ipotesi ${ }^{176}$. Oltre alla notizia fornita da Ibn Hayyan e alle ceramiche di provenienza nord-africana, siciliana e ispanica utilizzate come elementi decorativi nelle chiese medievali ${ }^{177}$, sono interessanti, per le relazioni commerciali degli arabi con la Sardegna, alcuni dati emersi recentemente negli scavi stratigrafici eseguiti ne Lu palazzu di Baldu (Luogosanto, OT), riguardanti dei manufatti vitrei ${ }^{178}$ che presentano una fascia di decorazione applicata in pasta vitrea blu, che lascia in negativo una decorazione epigrafica, forse originariamente dorata, in caratteri arabi ${ }^{179}$. Grazie al dato fornitoci da Ibn Hayyan ${ }^{180}$, relativo alla presenza a Cordova

\footnotetext{
${ }^{169}$ N. Silva, Marfiles, p. 269.

${ }^{170}$ D. Anedda, Bronces zoomorfos.

${ }^{171}$ P. Chalmeta, La Méditerranée, p. 342.

172 Ibidem.

${ }^{173}$ M. Amari, Biblioteca, vol. I, p. 25.

${ }^{174}$ C. Zedda, Bisanzio, p. 82.

${ }^{175}$ E. Ashtor, Ma'din.

${ }^{176}$ C. Renzi, I rapporti, s.p.

177 M.L. Ferru, I bacini.

${ }^{178}$ F. Pinna, Le testimonianze, pp. 29-30.

179 Ibidem, p. 29.

${ }^{180}$ P. Chalmeta, La Méditerranée, p. 342.
} 
di un messaggero del signore di Sardegna, accompagnato da alcuni mercanti amalfitani, si può ipotizzare che tramite questi scambi commerciali circolassero manufatti di gran pregio provenienti dal mondo arabo, quali stoffe, avori e metalli. È quindi pensabile che il commercio fosse bilaterale.

L'acquamanile della Pinacoteca Nazionale di Cagliari fu probabilmente fuso tra la fine del X e gli inizi dell'XI secolo, in una zona geografica della Spagna non facilmente identificabile, che solo per motivi commerciali potrebbe essere una regione del levante peninsulare.

\section{BIBLIOGRAFIA CITATA}

Abbas, Mohamed, The Fatimids (969-1171), in O'Kane, Bernard (ed.), The Treasures of Islamic Art in the Museums of Cairo, Cairo, American University in Cairo Press, 2006, pp. 46-91.

Allan, James; Ezzy, Waffiya; Scarce, Jennifer, Metalwork. Catalogue, in Jones, Dalu; Mitchell, George (eds.), The arts of Islam, catalogo della mostra (Londra, 8 Aprile-4 luglio), Londra, The Arts Council of Great Britain, 1976, pp. 157-204.

Amari, Michele, Biblioteca Arabo-Sicula, Torino-Roma, Loescher, 1881.

Amato, Pietro, Note orientative sui riti della Chiesa d'oriente e d'occidente, in Montevecchi, Benedetta; Vasco Rocca, Sandra (eds.), Suppellettile ecclesiastica. Dizionari terminologici, Firenze, Centro Di, 1988, vol. I, pp. 21-22.

Andrieu, Michel, Les Ordines romani du haut Moyen âge, Lovanio, Peeters, 1956-1960, 4 voll.

Anedda, Damiano, Arte mozarabica: storia degli studi e nuovi percorsi di lettura, in Cadeddu, Maria Eugenia; Mele, Maria Grazia (eds.), Frontiere del Mediterraneo, Seminario Internazionale di Studi (Cagliari, 10-12 ottobre 2002), 2003, pp. 149-170.

Anedda, Damiano, Bronces zoomorfos islámicos en Italia, in 711: El arte entre la hégira y el califato omeya de al-Andalus, atti delle V Jornadas Complutenses de Arte Medieval, Madrid, 16-18 novembre 2011 (in corso di stampa).

Anedda, Damiano, Bronzi islamici. Sculture zoomorfe medievali nei musei italiani, Roma, Artemide, 2012.

Arbeiter, Achim; Noack-Haley, Sabine, Christliche Denkmäler des frühen Mittelalters, Hispania Antiqua, vol. VIII, Magonza, Philipp von Zabern, 1999.

Ashtor, Eliyahu, Ma'din. I. Aspects économiques, in Enciclopédie de l'Islam, Nouvelle Édition, Leida, Brill, 1986, vol. V, pp. 969-970. 
Baer, Eva, Ibrīk, in Enciclopédie de l'Islam. Nouvelle Édition, Leida, Brill, 1982, Supplément livraison voll.V-VI, pp. 406-407.

Baer, Eva, Metalwork in Medieval Islamic Art, Albany, State of New York University Press, 1983.

Baldini Lippolis, Isabella, L'oreficeria nell'impero di Costantinopoli tra IV e VII secolo, Bari, Edipuglia, 1999.

Ballian, Anna, Three medieval Islamic brasses and the Mosul tradition of in-

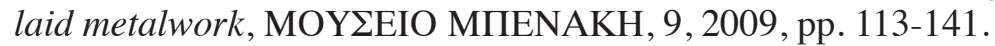

Ballian, Anna, A Singular Gift: an Islamic Predatory Bird at Mount Sinai, "Sinaiticus, The Bulletin of the Saint Catherine foundation" (2012), pp. 14-18.

Ballian, Anna, Bird-shaped vessel, in Evans, Helen C.; Ratliff, Brandie (eds.), Byzantium and Islam: Age of Transition, 7th-9th Century, New York, Metropolitan Museum of Art, 2012, núm. 38, pp. 64-66.

Baracchini, Clara; Bertelli, Carlo; Caleca, Antonino; Collareta, Marco; Dalli Regoli, Gigetta; Filieri, Maria Teresa (eds.), Lucca e l'Europa. Un'idea di medioevo. V-IX secolo, catalogo della mostra (Lucca, 25 settembre 2010-9 gennaio 2011), Lucca, Fondazione Ragghianti studi sull'arte, 2010.

Barrucand, Marianne (ed.), L'Égypte fatimide. Son art et son Histoire, Parigi, Presses de l'Université de Paris-Sorbonne, 1999.

Barin, Luigi Rodolfo, Catechismo liturgico, Rovigo, Industrie grafiche italiane, 1921.

Bautier, Robert-Henri, Datation et provenance $d u$ «paon aquamanile» du Louvre à l'inscription bilingue, latine et arabe, "Bulletin de la Société nationale des antiquaires de France" (1977), pp. 92-101.

Berlekamp, Persis, From Iraq to Fars: tracking cultural transformation in the 1322 Quazīn $\bar{\imath}$ 'Ajā'ib Manuscript, in Contadini, Anna (ed.), Arab painting. Text and image in illustrated arabic manuscripts, Leida, Brill, 2007, pp. 73-91.

Bernardini, Michele, Bronzo. Islam, in Enciclopedia dell'Arte Medievale, Roma, Istituto della Enciclopedia Italiana, 1992, vol. III, pp. 782-790.

Bernus-Taylor, Marthe (ed.), Les Andalousies. De Damas à Cordoue, catalogo della mostra (Parigi, 28 novembre 2000-15 aprile 2001), Parigi, Institut du Monde Arabe, 2000.

Bernus-Taylor, Marthe, Oggetti, mobili e arredi dalla conquista araba all'invasione mongola, in Chuvin, Pierre (ed.), Le arti in Asia centrale, Milano, Garzanti, 2002, pp. 359-393.

Bloch, Peter, Acquamanili. Oggetti medievali per uso sacro e profano, Milano, F.M. Ricci, 1982. 
Bloom, Jonathan M., Arts of the City Victorious. Islamic Art and Architecture in Fatimid North Africa and Egypt, Cairo, The American University in Cairo Press, 2008.

Bradshaw, Paul F., Alle origini del culto cristiano. Fonti e metodi per lo studio della liturgia dei primi secoli, Monumenta Studia Instrumenta Liturgica, vol. XLVI, Città del Vaticano, Libreria Editrice Vaticana, 2007.

Braun, Joseph, Das christliche Altargerät in seinem Sein und in seiner Entwicklung, Monaco di Baviera, Max Hueber, 1932.

Bux, Nicola; Loconsole, Michele, I Misteri degli Orientali. I sacramenti bizantini comparati con la liturgia romana e i riti giudaici, Siena, Edizioni Cantagalli, 2006.

Caballero Zoreda, Luis; Rodríguez Trobajo, Eduardo; Murillo Fragero, José Ignacio; Martín Talaverano, Rrafael, Las iglesias asturianas de Pravia y Tuñón. Arqueología de la arquitectura, Anejos de Archivo Español de Arqueología, vol. LIV, Madrid, Consejo Superior de Investigaciones Científicas, 2010.

Cabrol, Fernand ; Leclerq, Henri, Dictionnaire d'archéologie chrétienne et de liturgie, Parigi, Librairie Letquezey et Ane, 1903-1953, 15 voll.

Callewaert, Camillus, De amictu, "Ephemerides Liturgicae, Commentarium cura et studio presbyterorum congregationis missionis alternis mensibus editum" 50/10 (1936), pp. 235-335.

Capra, Arnaldo, Inventari degli argenti, libri e arredi sacri delle chiese di Santa Gillia, di San Pietro e di Santa Maria di Cluso, "Archivio Storico Sardo" 3 (1907), pp. 420-426.

Casagrande, Vincenzo, L'arte al servizio della Chiesa, Torino, Società Editrice Internazionale, 1931, 2 voll.

Casamar, Manuel, Arqueta de Hišăm, in Dodds, Jerrilynn Denise (ed.), Al-Andalus. Las artes islámicas en España, catalogo della mostra (Granada, 18 marzo-7 giugno 1992, New York, 1 luglio-27 settembre 1992), Madrid, El Viso, 1992, núm. 9, pp. 208-209.

Chalmeta, Pedro, La Méditerranée occidentale et Al-Andalus de 934 à 941: les donnés d'Ibn Hayyân, "Rivista degli Studi Orientali" 50 (1976), pp. 337-351.

Charritat, Maguy, Lion à queue articulée, in Bernus-Taylor, Marthe (ed.), Arabesques et jardins de paradis. Collections françaises d'art islamique catalogo della mostra, (Parigi, 16 ottobre 1989-15 gennaio 1990), Parigi, Réunion des musées nationaux, 1989, núm. 127, p. 154.

Compareti, Matteo, The So-Called Senmurv in Iranian Art: A Reconsideration of an Old Theory, in Borbone, Pier Giorgio; Mengozzi, Alessandro ; Tosco, Mauro (eds.), Loquentes linguis. Studi linguistici e orientali 
in onore di Fabrizio A. Pennacchietti, Wiesbaden, Harrassowitz Verlag, 2006, pp. 185-200.

Contadini, Anna, Acquamanile in forma di uccello, in Curatola, Giovanni (ed.), Eredità dell'Islam. Arte islamica in Italia, catalogo della mostra (Venezia, 30 ottobre 1993-30 aprile 1994), Cinisello Balsamo, Silvana Editoriale, 1993, núm. 42, pp. 125-126.

Contadini, Anna, La Spagna dal II/VIII al VII/XIII secolo, in Curatola, Giovanni (ed.), Eredità dell'Islam. Arte islamica in Italia, catalogo della mostra (Venezia, 30 ottobre 1993-30 aprile 1994), Cinisello Balsamo, Silvana Editoriale, 1993, pp. 105-113.

Contadini, Anna, Quadrupede in bronzo, in Curatola, Giovanni (ed.), Eredità dell'Islam. Arte islamica in Italia, catalogo della mostra (Venezia, 30 ottobre 1993-30 aprile 1994), Cinisello Balsamo, Silvana Editoriale, 1993, n 41, pp. 124-125.

Contadini, Anna, Le arti del periodo fatimide, in Cassanelli, Roberto (ed.), Il Mediterraneo e l'arte nel Medioevo, Milano, Jaca Book, 2000, pp. 119-137.

Contadini, Anna, Le arti in Spagna e Marocco nei periodi almoravide e almoade, in Cassanelli, Roberto (ed.), Il Mediterraneo e l'arte nel Medioevo, Milano, Jaca Book, 2000, pp. 54-60.

Contadini, Anna; Camber, Richard; Northover, Peter, Beasts that roared: the Pisa Griffin and the New York Lion, in Ball, Warwick; Harrow, Leonard (eds.), Cairo to Kabul. Afghan and Islamic Studies Presented to Ralph Pinder-Wilson, Londra, Melisende, 2001, pp. 65-83.

Contadini, Anna, Translocation and transformation: some Middle Eastern Objects in Europe, in Saurma-Jeltsch, Lieselotte E.; Eisenbeiss, Anja (eds.), The Power of Things and the Flow of Cultural Transformations, Berlino, Deutscher Kunstverlag, 2010, pp. 42-64.

Coroneo, Roberto, Acquamanile a forma di pavone, in Serra, Renata, Pittura e scultura dall'età romanica alla fine del '500, Nuoro, Ilisso, 1990, $\mathrm{n}^{\mathrm{o}} 6, \mathrm{p} .24$.

Coroneo, Roberto, Il reliquiario di Santa Maria Navarrese e altre tracce materiali della presenza islamica in Sardegna, in Angelelli, Walter; Pomarici, Francesca, Forme e Storia. Scritti di arte medievale e moderna per Francesco Gandolfo, Rende, Artemide, 2011, pp. 119-125.

Cremascoli, Giuseppe La simbologia e teologia battesimali, in L'acqua nei secoli altomedievali. Settimane di studio della fondazione Centro Italiano di Studi sull'Alto Medioevo (Spoleto, 12-17 aprile 2007), Spoleto, Centro Italiano di Studi sull'Alto Medioevo, 2008, vol. LV-2, pp. 1147-1167.

Cruikshank Dodd, Erica, On a bronze rabbit from fatimid Egypt, "Kunst des Orients" 8 (1972), pp. 60-76. 
Curatola, Giovanni; Scarcia, Gianroberto, Le arti nell'Islam, 2 edizione, Urbino, Carocci, 2001.

Davillier, Jean Charles, Recherches sur l'orfévrerie en Espagne au Moyen âge et à la Renaissance, Parigi, A. Quintin, 1879.

De Longpérier, Adrien, Vase arabo-sicilien de l'œuvre de Salomon, "Revue Archéologique" 12 (1865), pp. 356-367.

Della Torre, Stefano; Marinelli, Massimo (eds.), Rationale Divinorum Officiorum. Durandi, Guillelmi. Liber I-III, traduzione italiana di Freguglia, Gian Franco, in Monumenta Studia Instrumenta Liturgica, vol. XIV, Città del Vaticano, Libreria Editrice Vaticana, 2001.

Discussione sulla lezione Cremascoli, in L'acqua nei secoli altomedievali. Settimane di studio della fondazione Centro Italiano di Studi sull'Alto Medioevo (Spoleto, 12-17 aprile 2007), Spoleto, Centro Italiano di Studi sull'Alto Medioevo, 2008, vol. LV-2, pp. 1169-1172.

Dodds, Jerrilynn Denise (ed.), Al-Andalus. Las artes islámicas en España, catalogo della mostra (Granada, 18 marzo-7 giugno 1992, New York, 1 luglio-27 settembre 1992), Madrid, El Viso, 1992.

Du Cange, Charles, Ciminile, in Glossarium Mediae et Infimae Latinitatis, Parigi, Excudebant Firmin Didot Fratres, 1842, vol. II, p. 351.

Duchesne, Louis (ed.), Le Liber Pontificalis. Texte, introduction et commentaire, reimpression conforme à l'edition de 1955, Parigi, De Boccard, 1981, vol. I.

Evans, Helen C.; Ratliff, Brandie (eds.), Byzantium and Islam: Age of Transition, 7th-9th Century, New York, Metropolitan Museum of Art, 2012.

Fagiolo, Marcello; Madonna, Maria Luisa (eds.), Roma 1300-1875. L'arte degli anni santi, catalogo della mostra (Roma, Palazzo Venezia, 20 dicembre 1984-6 aprile 1985), Milano, Mondadori, 1984.

Ferru, Maria Laura, I bacini ceramici delle chiese medievali: ricordo della mancata invasione araba della Sardegna, in I bacini murati medioevali. Problemi e stato della ricerca, atti del XXVI convegno internazionale della ceramica (Albisola, 28-30 maggio 1993), Firenze, All'insegna del Giglio, 1996, pp. 287-295.

Fioramonti, Stanislao (ed.), Innocenzo III. Il sacrosanto Mistero dell'Altare, in Monumenta Studia Instrumenta Liturgica, vol. XV, Città del Vaticano, Libreria Editrice Vaticana, 2002.

Glück, Heinrich; Diez, Ernst, Arte del Islam, Barcellona, Labor, 1961.

Gómez-Moreno Martínez, Manuel, Iglesias Mozárabes. Arte español de los siglos IX a XI, Madrid, Junta para Ampliación de Estudios e Investigaciones Científicas - Centro de Estudios Históricos, 1919. 
Gómez-Moreno Martínez, Manuel, El arte árabe español hasta los almohades. Arte mozárabe, Ars Hispaniae. Historia Universal del Arte Hispánico, vol. III, Madrid, Plus-Ultra, 1951.

González Navarro, Carlos, Testamentaría e inventario de bienes de Mariano Fortuny en Roma, "Locus amoenus" 9 (2007-2008), pp. 319-349.

Grabar, Oleg, Arte islamica. Formazione di una civiltà, Milano, Electa 1989.

Grabar, Oleg, About a bronze bird, in Sears, Elizabeth; Thomas, Thelma Katrina, Reading Medieval Images. The Art Historian and the Object, Ann Arbor, University of Michigan Press, 2002, pp. 117-125.

Hendrie, Robert (ed.), An essay upon various art in three books by Theophilus called also Rugerus, priest and monk, Londra, George Woodfall and son, 1847.

Inglisa, Rosalinda, Arte islamica del metallo: gli acquamanili e il pavone della Pinacoteca Nazionale di Cagliari, tesi di laurea, Università degli Studi di Cagliari, Facoltà di Lettere e Filosofia, anno accademico 2004-2005.

Kröger, Jens, Sasanidischer Stuckdekor, Magonza, Philipp von Sabern, 1982.

Kühnel, Ernst, Maurische Kunst, Berlino, Cassires Verlag, 1924.

Labarte, Jules, Histoires des arts industriels au Moyen âge et á l'époque de la Renaissance, Parigi, Librairie de A. Morel, 1864-1866, 4 voll.

Lévi-Provençal, Évariste, Decadencia y caída del califato de Córdoba, in Menéndez Pidal, Ramón (ed.), Historia de España. España musulmana hasta la caída del Califato de Córdoba (711-1031 de J.C.), IV, Madrid, Espasa-Calpe, 1987, pp. 455-489.

Liccardo, Giovanni, Architettura e liturgia nella chiesa antica, Milano, Skira, 2005.

Little, Charles T., Leone, in Maria Andaloro (ed.), Nobiles Officinae. Perle, filigrane e trame di seta dal Palazzo Reale di Palermo, catalogo della mostra (Palermo, 17 dicembre 2003-10 marzo 2004; Vienna, 30 marzo-13 giugno 2004), Catania, Giuseppe Maimone, 2006, $\mathrm{n}^{\circ}$ II.19, pp. 154-157.

Longoni, Francesco, Ambrosiana. Liturgia, in Enciclopedia Cattolica, Firenze, Sansoni, 1948, vol. I, pp. 1008-1114.

Makariou, Sophie, Aiguière, in Bernus-Taylor, Marthe (ed.), Les Andalousies. De Damas à Cordoue, catalogo della mostra (Parigi, 28 novembre 2000-15 aprile 2001), Parigi, Institut du Monde Arabe, 2000, no 168, p. 151.

Makariou, Sophie, Aquamanile en forme de paon, in Bernus-Taylor, Marthe (ed.), Les Andalousies. De Damas à Cordoue, catalogo della mostra (Parigi, 28 novembre 2000-15 aprile 2001), Parigi, Institut du Monde Arabe, 2000, $\mathrm{n}^{\circ}$ 87, p. 111. 
Makariou, Sophie, Aquamanile en forme de paon, in Bernus-Taylor, Marthe (ed.), Les Andalousies. De Damas à Cordoue, catalogo della mostra (Parigi, 28 novembre 2000-15 aprile 2001), Parigi, Institut du Monde Arabe, 2000, n 88, p. 112.

Makariou, Sophie, Bouche de fontaine en forme de cerf, in Bernus-Taylor, Marthe (ed.), Les Andalousies. De Damas à Cordoue, catalogo della mostra (Parigi, 28 novembre 2000-15 aprile 2001), Parigi, Institut du Monde Arabe, 2000, no 90, p. 114.

Maltese, Corrado; Serra, Renata, Episodi di una civiltà anticlassica, in Barreca, Ferruccio (ed.), Sardegna, Electa, Venezia, 1969, pp. 117-408.

Maryon, Herbert, La lavorazione dei metalli. Oreficeria, argenteria e tecniche complementari, Milano, Hoepli, 1998.

Melikian-Chirvani, Assadullah Souren, Les bronzes du Khorasan I, "Studia Iranica" 3/1 (1974), pp. 29-50.

Melikian-Chirvani, Assadullah Souren, Les bronzes du Khorasan II, "Studia Iranica" 4/1 (1975), pp. 51-71.

Melikian-Chirvani, Assadullah Souren, Les bronzes du Khorasan III, "Studia Iranica" 4/2 (1975), pp. 187-205.

Melikian-Chirvani, Assadullah Souren, Les bronzes du Khorasan IV, "Studia Iranica" 5/2 (1976), pp. 203-212.

Melikian-Chirvani, Assadullah Souren, Les bronzes du Khorasan V, "Studia Iranica" 6/2 (1977), pp. 185-210.

Melikian-Chirvani, Assadullah Souren, Les bronzes du Khorasan VI, "Studia Iranica" 7 (1978), pp. 7-31.

Mende, Ursula, Acquamanile, in Enciclopedia dell'Arte Medievale, Roma, Istituto della Enciclopedia Italiana, 1991, vol. I, pp. 102-105.

Mesina, Salvatorangelo Maria, L'acqua santa nel battesimo, nel sacrificio eucaristico e in alcuni riti minori. Storia. Simbolismo, in Dissertationes ad lauream, vol. XXXVII, Pontificia Facultas Theologica S. Bonaventurae, Ordinis Fratrum Minorum Conventualium in Urbe, Roma, Miscellanea Francescana, 1957.

Migeon, Gaston, Manuel d'Art Musulman. Arts Plastiques et Industrielles, Parigi, Picard, 1927.

Migne, Jacques Paul (ed.), Honorii Augustodunensis, Operum, pars tertia, Liturgica, Speculum Ecclesiae, Dominica in Palmis, Patrologia Latina, vol. CLXXII, Lutetiae Parisiorum, 1853.

Montevecchi, Benedetta; Vasco Rocca, Sandra (eds.), Suppellettile ecclesiastica. Dizionari terminologici, Firenze, Centro Di, 1988.

Orbeli, Josef, Sassanian and Early Islamic Metalworks, in Pope, Arthur Upham (ed), A survey of Persian art, Oxford-Londra, Oxford University Press, 1938, vol. I, pp. 716-770. 
Overlaet, Bruno (ed.), Splendeur des Sassanides. L'empire Perse entre Rome et la Chine (224 - 642), catalogo della mostra (Bruxelles, 12 febbraio-25 aprile 1993), Bruxelles, Crédit communal, 1993.

Pala, Andrea, Arredo liturgico medievale. La documentazione scritta e materiale in Sardegna fra IV e XIV secolo, Cagliari, AV, 2011.

Pala, Andrea, Paramenti sacri e suppellettili ecclesiastiche nell'inventario duecentesco di Santa Gilla, San Pietro e Santa Maria di Cluso, "Theologica \& Historica. Annali della Pontificia Facolta Teologica della Sardegna" 19 (2010), pp. 359-400.

Gayangos, Pascual de (ed.), Ahmed ibn Mohammed al-Maqqari, The history of the Mohammedan dynasties in Spain. Extracted from the Nafhut-tib min ghosni-l-Andalusi-r-rattib wa tarikh lisanu-d-din Ibni-lKhattib, vol. I, Londra 1840-1843.

Papadopulo, Alexandre, L'Islam e l'arte musulmana, Milano, Garzanti, 1992

Penco, Gregorio, Il monachesimo fra spiritualità e cultura, Milano, Jaca Book, 1991.

Pinell, Jordi, Liturgia Hispánica, in Aldea Vaquero, Quintín; Marín Martínez, Tomas; Vives, José (eds.), Diccionario de Historia Eclesiástica de España, Madrid, Consejo Superior de Investigaciones Científicas Instituto Enrique Flórez, 1972, pp. 1303-1320.

Pinna, Fabio, Le testimonianze archeologiche relative ai rapporti tra gli Arabi e la Sardegna nel medioevo, "RiMe. Rivista dell'Istituto di Storia dell'Europa Mediterranea" 4 (2010), pp. 11-37.

Piras, Sara Silvia; Dessì, Gisa (eds.), Il registro di San Pietro di Sorres, Cagliari, Centro di studi filologici sardi - CUEC, 2003.

Renzi Rizzo, Catia, I rapporti diplomatici tra il re Ugo di Provenza e il califfo 'Abd ar-Rahmân III: fonti cristiane e fonti arabe a confronto (http:// www.rmojs.unina.it/index.php/rm/article/view/261/253) [consultato: $16 / 02 / 2012]$.

Righetti, Mario, Manuale di Storia liturgica, vol. I, Introduzione generale, Milano, Ancora, 1945.

Righetti, Mario, Manuale di Storia liturgica, vol. II, L'anno liturgico, il breviario, Milano, Ancora, 1946.

Robinson, Cynthia, Grifo de Pisa, in Dodds, Jerrilynn Denise (ed.), Al-Andalus. Las artes islámicas en España, catalogo della mostra (Granada, 18 marzo-7 giugno 1992, New York, 1 luglio-27 settembre 1992), Madrid, El Viso, 1992, pp. 216-218, n 15.

Rocha, Pedro, Liturgia della cappella papale, liturgia dei frati Minori e liturgia dei frati Predicatori, in Boyle, Leonard E.; Gy, Pierre-Marie (eds.), Aux origines de la liturgie dominicane. Le manuscrit Santa Sabina XIV L 1, Collection de École Française de Rome, 327, Do- 
cuments, études et répertoire publiés par l'Institute de recherche et d'histoire teste, 679, Parigi-Roma, CNRS Éditions - École Française de Rome, 2004, pp. 115-125.

Rogers, John Michael, Acquamanile in the form of a goose, in Rogers, John Michael, The Arts of Islam. Treasures from the Nasser D Khalili Collections, catalogo della mostra (New South Wales, 22 giugno-23 settembre 2007), Sydney, Art Gallery of New South Wales, 2007, pp. $78-79, \mathrm{n}^{\circ} 68$.

Rohault de Fleury, Charles, La messe. Études archéologiques sur les monuments, Parigi, Librairies-imprimeries reunies, 1883-1889, 8 voll.

Sangiorgi, Silvia, L'iconografia paleocristiana, in Martorelli, Rossana, Archeologia cristiana medievale in Sardegna. Introduzione allo studio, Cagliari, CUEC, 2008, pp. 102-114.

Scerrato, Umberto, Metalli islamici, Milano, Fabbri, 1966.

Scerrato, Umberto, Arte islamica in Italia, in Gabrieli, Francesco; Scerrato, Umberto, Gli arabi in Italia. Cultura, contatti e tradizioni, Milano, Scheiwiller - Credito italiano, pp. 271-571.

Serra, Renata, L'oratorio delle anime a Massama, "Annali delle Facoltà di Lettere, Filosofia e Magistero dell’Università di Cagliari” 26 (1971), pp. 33-68.

Serra, Renata, Le raccolte sconosciute del Museo Nazionale di Cagliari, “Almanacco di Cagliari" (1976), s.p.

Serra, Renata, Pittura e scultura dall'età romanica alla fine del '500, Nuoro, Ilisso 1990.

Serreli, Marcella, Acquamanile, in Porcella, Maria Francesca; Serreli, Marcella; Degioannis, Luisa; Maxia, Antonia Giulia, Moriscos. Echi della presenza e della cultura islamica in Sardegna, catalogo della mostra (Cagliari, 1993), Cagliari, Pinacoteca Nazionale di Cagliari, 1993, $\mathrm{n}^{\circ} 49$, p. 45 .

Shalem, Avinoam, Islam Christianized. Islamic Portable Objects in the Medieval Church Tresuries of the Latin West, Francoforte, Peter Lang, 1998.

Shepherd, Dorothy, Two silver Ryta, "Bulletin of the Cleveland Museum of Art", 53 (1966) pp. 289-317.

Siddi, Lucia, Acquamanile a forma di volatile, in Pinacoteca Nazionale di Cagliari, Muros, Soprintendenza ai beni ambientali architettonici artistici e storici - Credito Industriale Sardo, 1988, vol. I, p. 129.

Silva, Romano, La Basilica di San Frediano a Lucca. Immagine Simbolica di Roma Cristiana, Lucca, Maria Pacini Fazzi Editore, 2010.

Silva Santa-Cruz, Noelia, Marfiles, in Eloy Momplet Míguez, Antonio, El arte hispanomusulmán, Madrid, Encuentro, 2008, pp. 244-277. 
Siniscalco, Paolo, Le antiche chiese orientali. Storia e letteratura, Roma, Città Nuova Editrice, 2005.

Stasolla, Maria Giovanna, Il collezionismo di arte islamica fra Italia e Spagna nel XIX secolo. Il caso di Mariano Fortuny y Marsal, in Beltrán Fortes, José; Cacciotti, Beatrice; Palma, Beatrice (eds.), Arqueología, coleccionismo y antigüedad: España e Italia en el siglo XIX, Siviglia, Universidad de Sevilla, 2007, pp. 661-685.

Swarzenski, Georg, Samson killing the lion. A medieval bronze group, "Bulletin of the Boston Museum of Fine Arts" 38 (1940), pp. 67-74.

Torres Balbás, Leopoldo, Arte hispanomusulmán hasta la caída del califato de Córdoba, in Menéndez Pidal, Ramón (ed.), Historia de España. España musulmana hasta la caída del Califato de Córdoba (7111031 de J.C.). Instituciones y vida social e intelectual, V, Madrid, Espasa-Calpe, 1987, (5 ed.), pp. 331-788.

Trésors d'art du moyen âge en Italie, catalogo della mostra (Parigi, maggioluglio 1952), Parigi, Les Presses Artistiques, 1952.

Turtas, Raimondo, Il registro di Sorres come fonte storica, in Piras, Sara Silvia; Dessì, Gisa (eds.), Il registro di San Pietro di Sorres, Cagliari, Centro di studi filologici sardi - CUEC, 2003, pp. VII-XXXV.

Vílchez Vílchez, Carlos (ed.), Las lámparas de Medina Elvira, catalogo della mostra (Granada, maggio 2003), Granada, Consejería de Cultura de la Junta de Andalucía - Fundación Caja Rural de Granada, 2003.

Von Gladiss, Almut, Figure de cerf, in Trésors fatimides du Caire, catalogo della mostra (Parigi, 28 aprile- 30 agosto 1998), Parigi, Institut du Monde Arabe, 1998, n 49, pp. 120-121.

Ward, Rachel; La Niece, Susan; Hook, Duncan; White, Raymond, Veneto-Saracenic metalwork: an analysis of the bowls and incense burners in the British Museum, in Hook, Duncan; Gaimster, David R.M. (eds.), Trade and discovery: the scientific study of artefacts from post-medieval Europe and beyond Trade and discovery, Londra, British $\mathrm{Mu}-$ seum, 1995, pp. 235-258.

Ward, Rachel, Acquamanile a forma di falco, in Baracchini, Clara; Bertelli, Carlo; Caleca, Antonino; Collareta, Marco; Dalli Regoli, Gigetta; Filieri, Maria Teresa (eds.), Lucca e l'Europa. Un'idea di medioevo. V-IX secolo, catalogo della mostra (Lucca, 25 settembre 2010-9 gennaio 2011), Lucca, Fondazione Ragghianti studi sull'arte, 2010, n ${ }^{\circ}$ 91, pp. 198-201.

Zagari, Francesca, Approvvigionamento e lavorazione delle leghe di rame a Costantinopoli tra XI e XII secolo, in Iacobini, Antonio (ed.), Le porte del Paradiso. Arte e tecnologia bizantina tra Italia e Mediterraneo, Roma, Campisano, 2009. 
Zanotti, Maria Gabriella, Ss. Gervasius et Protasius. Titulus, in Steinby, Eva Margareta, Lexicon Topographicum Urbis Romae, Roma, Quasar, 1995, vol. II, p. 371.

Zedda, Corrado, Bisanzio, l'Islam e i giudicati: la Sardegna e il mondo mediterraneo tra VII e XI secolo, "Archivio Storico Giuridico Sardo di Sassari” 10 (2006), pp. 39-112.

Zichi, Giancarlo, Sorres e la sua diocesi, Sassari, Fondazione Collegium Mazzotti, 1975.

Zozaya, Juan, Lamp à deux becs avec l'inscription oc opus Salomonis erat, in Bernus-Taylor, Marthe (ed.), Les Andalousies. De Damas à Cordoue, catalogo della mostra (Parigi, 28 novembre 2000-15 aprile 2001), Parigi, Institut du Monde Arabe, 2000, n 95, p. 117.

Fecha de recepción del artículo: febrero 2012

Fecha de aceptación y versión final: febrero 2013 